\title{
CASSON-TYPE INVARIANTS IN DIMENSION FOUR
}

\author{
DANIEL RUBERMAN AND NIKOLAI SAVELIEV
}

\section{INTRODUCTION}

This article surveys our ongoing project about the relationship between invariants extending the classical Rohlin invariant of homology spheres and those coming from 4-dimensional (Yang-Mills) gauge theory; it will appear in the Proceedings of the Fields-McMaster Conference on Geometry and Topology of Manifolds. We are mainly concerned with a special class of manifolds for which the two types of invariants are defined and can be compared. This class contains, in particular, manifolds having the homology of $S^{1} \times S^{3}$. Rohlin's theorem about the signature of closed smooth spin 4-manifolds gives rise to a mod-2 invariant of a homology $S^{1} \times S^{3}$. On the gauge theoretic side, the invariant is obtained by counting flat connections on appropriate bundles. This count is inspired by Donaldson's 13 count of anti-self-dual connections on $S U(2)$ bundles; its flat analogue was first studied by Furuta and Ohta [21].

The main conjecture towards which this project is directed is that the Rohlin invariant and the gauge theoretic invariant coincide for homology $S^{1} \times S^{3}$. The model for the whole discussion is Casson's beautiful theorem relating his invariant (in its gauge theoretic manifestation as described by Taubes [4]) and Rohlin's invariant of homology 3-spheres. We will discuss the implications of this conjecture for some classical problems in low-dimensional topology, and progress we have made towards proving the conjecture. This progress includes the verification of the conjecture in some special cases, a 'surgery' program for approaching the conjecture by expanding its scope to include a wider category of manifolds, and the verification of this expanded conjecture for homology 4-tori. Much of this material is contained in our three papers [36, 37, 38, but we have included a broader overview as well as some additional examples.

Acknowledgments. We would like to thank Scott Baldridge for pointing out the manifolds described in Section [8, and Liviu Nicolaescu for his input on computing orientations of flat moduli spaces over these manifolds. We also

2000 Mathematics Subject Classification. 57M27, 57R58, 58D27.

The first author was partially supported by NSF Grants 9971802 and 0204386 . The second author was partially supported by NSF Grant 0305946 and Max Planck Institut für Mathematik in Bonn. 
thank Kim Frøyshov for some interesting conversations related to the material presented in this paper. We express our appreciation to the conference organizers for providing such a stimulating environment at McMaster.

\section{The ROHLIN InVARIANT}

This section is a review of the homology cobordism group and the Rohlin invariant and some of their applications in topology. More information and references may be found in the books 43,42 .

2.1. Homology spheres. By an (integral) homology sphere we will mean a closed oriented $3-$ manifold $\Sigma$ such that $H_{*}(\Sigma ; \mathbb{Z})=H_{*}\left(S^{3} ; \mathbb{Z}\right)$. According to the Poincaré conjecture, every simply connected homology sphere is homeomorphic to $S^{3}$. There exist, however, many non-simply connected homology spheres, as the following examples demonstrate.

For any three positive pairwise relatively prime integers $p, q$, and $r$, the zero set of the complex polynomial $x^{p}+y^{q}+z^{r}$ is a complex surface which has an isolated singularity at the origin. The link of this singularity,

$$
\Sigma(p, q, r)=\left\{x^{p}+y^{q}+z^{r}=0\right\} \cap S^{5},
$$

is a homology sphere sphere referred to as a Brieskorn homology sphere. The Brieskorn homology sphere $\Sigma(2,3,5)$ is also known as the Poincaré sphere. The above construction can be generalized to obtain Seifert fibered homology spheres $\Sigma\left(a_{1}, \ldots, a_{n}\right)$ for any positive pairwise relatively prime integers $a_{1}, \ldots, a_{n}$.

A more general construction of homology spheres is as follows. Let $k$ be a knot in $S^{3}$ and $q$ an integer then any manifold $S^{3}+(1 / q) k$ obtained by $(1 / q)$-surgery of $\Sigma$ along $k$ is a homology sphere. For example, $\Sigma(2,3,5)$ can be obtained by $(-1)$-surgery on the left handed trefoil. Not all homology spheres can be obtained by this construction; however, if one allows surgery along links of more than one component, one obtains all homology spheres (and in fact all closed oriented 3-manifolds).

2.2. Homology cobordisms. A homology cobordism from a homology sphere $\Sigma_{0}$ to a homology sphere $\Sigma_{1}$ is a smooth compact oriented 4-manifold $W$ with boundary $\partial W=-\Sigma_{0} \cup \Sigma_{1}$ such that the inclusions $\Sigma_{i} \rightarrow W$ induce isomorphisms $H_{*}\left(\Sigma_{i} ; \mathbb{Z}\right) \rightarrow H_{*}(W ; \mathbb{Z})$ for $i=0,1$.

An obvious example of a homology cobordism from a homology sphere $\Sigma$ to itself is the product $W=[0,1] \times \Sigma$. The mapping cylinder $W_{\tau}$ of any orientation preserving diffeomorphism $\tau: \Sigma \rightarrow \Sigma$ is also a homology cobordism from $\Sigma$ to itself; note that $W_{\tau}$ is diffeomorphic to the product $[0,1] \times \Sigma$ but need not be diffeomorphic to it rel boundary.

The first example of a non-trivial homology cobordism was constructed by Mazur; his manifold is a simply connected homology cobordism between $S^{3}$ and $\Sigma(2,5,7)$. Many more examples of homology cobordisms can be obtained by performing surgery along knot and link concordances in $[0,1] \times$ $S^{3}$, and by various other constructions. 
2.3. Homology cobordism group. The relation of being homology cobordant is an equivalence relation on the class of homology spheres. The set of equivalence classes of homology spheres with the operation induced by connected sum is an abelian group called the homology cobordism group and denoted $\Theta^{3}$. The inverse element of $[\Sigma] \in \Theta^{3}$ is $[-\Sigma]$, and the zero element is the homology cobordism class of $S^{3}$.

2.4. The Rohlin invariant. Every homology sphere $\Sigma$ is the boundary of a smooth compact spin 4-manifold $X$, whose signature is necessarily divisible by eight. Additivity of the signature and the Rohlin theorem (which asserts that the signatures of any two such manifolds bounding $\Sigma$ can only differ by a multiple of sixteen) imply that the quantity

$$
\rho(\Sigma)=\frac{1}{8} \operatorname{sign} X \quad(\bmod 2)
$$

only depends on $\Sigma$ and takes values in $\mathbb{Z}_{2}=\mathbb{Z} / 2 \mathbb{Z}$. It is called the Rohlin invariant of $\Sigma$. One can easily see that $\rho$ defines a homomorphism $\rho: \Theta^{3} \rightarrow$ $\mathbb{Z}_{2}$.

As an example, consider the singularity at zero of $x^{2}+y^{3}+z^{5}=0$. It has a resolution $X$ which is a smooth compact simply connected spin manifold with boundary $\Sigma(2,3,5)$ and intersection form $E_{8}$. Therefore, sign $X=-8$ and $\rho(\Sigma(2,3,5))=1(\bmod 2)$. In particular, we see that $\rho: \Theta^{3} \rightarrow \mathbb{Z}_{2}$ is an epimorphism.

The Rohlin invariant can be defined more generally for pairs $(Y, \sigma)$, where $Y$ is a closed oriented 3-manifold and $\sigma$ is a spin structure on $Y$. By definition,

$$
\rho(Y, \sigma)=\frac{1}{8} \operatorname{sign} X \quad(\bmod 2),
$$

where $X$ is any smooth compact spin 4 -manifold with (spin) boundary $(Y, \sigma)$. This invariant takes values in $\mathbb{Q} / 2 \mathbb{Z}$. A homology sphere $\Sigma$ has a unique spin structure hence it does not show up in the notation $\rho(\Sigma)$.

2.5. The structure of $\Theta^{3}$. The fact that $\rho: \Theta^{3} \rightarrow \mathbb{Z}_{2}$ is an epimorphism had been the only known general fact about $\rho$ and $\Theta^{3}$ until the early 1980's when some progress was made using gauge theory. The Donaldson diagonalizability theorem for the intersection forms of smooth closed oriented definite 4 -manifolds implies right away that $\Theta^{3}$ is an infinite group. In fact, one can see that $\Sigma(2,3,5)$ is an element of infinite order in $\Theta^{3}$. More elements of infinite order were found by Fintushel and Stern using equivariant gauge theory. Later Furuta showed that $\Theta^{3}$ is infinitely generated - in fact, Brieskorn homology spheres $\Sigma(2,3,6 m-1)$ with $m \geq 1$ all have infinite order in $\Theta^{3}$ and are linearly independent over $\mathbb{Z}$.

The question of whether $\Theta^{3}$ has torsion remains open; the latest result in this direction is as follows, see [19, 20] and 41. 
Theorem 2.1. Let $\Sigma$ be a homology sphere which is homology cobordant to a Seifert fibered homology sphere. If $\rho(\Sigma)$ is non-trivial then $\Sigma$ has infinite order in $\Theta^{3}$.

It is not known if all homology spheres are homology cobordant to Seifert fibered ones but this is considered highly unlikely. In particular, Frøyshov has an (unpublished) extension of his work on the $h$-invariant 18, which seems to give rise to counterexamples.

Describing the structure of $\Theta^{3}$ is interesting in its own right but also because of its applications some of which are described below, see also Section 4.8 .

2.6. Triangulation conjecture. The triangulation conjecture in dimension $n$ asserts that every (closed) topological $n$-manifold is homeomorphic to a simplicial complex. This conjecture has been long known to hold in dimensions three and lower. It fails in dimension four, which follows by combining Freedman's classification of simply connected topological manifolds and the Casson invariant theory, see Section 3.5

The triangulation conjecture remains open in dimensions $n \geq 5$. Amazingly enough, it is equivalent in these dimensions to a specific question about the structure of $\Theta^{3}$. Namely, according to a theorem of Matumoto [30] and Galewski and Stern [22], the triangulation conjecture holds in all dimensions $n \geq 5$ if and only if there exists a homology sphere $\Sigma$ of order two in $\Theta^{3}$ having non-trivial Rohlin invariant. Needless to say, no such homology sphere has been found; Theorem 2.1 implies that there is no need to search among Seifert fibered homology spheres and the homology spheres homology cobordant to them.

2.7. Simply connected homology cobordisms. Every homology sphere $\Sigma$ is homology cobordant to itself via the product homology cobordism $[0,1] \times \Sigma$. This cobordism is not simply connected unless $\Sigma$ is. A natural question arises whether $\Sigma$ can be homology cobordant to itself via a simply connected homology cobordism. For some homology spheres the answer to this question is positive, while for others it is negative. For example, there are no simply connected homology cobordisms of the Poincaré homology sphere to any homology sphere, not just to itself, see Taubes [4]. More examples of such behavior have been given by Fintushel and Stern [15.

It has been conjectured that no two homology spheres with non-trivial Rohlin invariant can be homology cobordant via a simply connected homology cobordism (note that the examples of Fintushel and Stern may have both trivial and non-trivial Rohlin invariants).

\section{The Casson invariant}

The Casson invariant is an integer valued invariant of homology spheres, defined by Casson in 1985. For a homology sphere $\Sigma$, it was defined as a 'creative' count of $S U(2)$ representations of $\pi_{1} \Sigma$, see Akbulut-McCarthy [3]. 
Later, Taubes 48] reformulated this original definition in gauge theoretic terms using the fact that every $S U(2)$ representation of $\pi_{1} \Sigma$ arises as the holonomy representation of a flat connection.

3.1. Definition of the Casson invariant. Let $P \rightarrow \Sigma$ be a trivialized $S U(2)$ bundle over a homology sphere $\Sigma$ and consider the moduli space $\mathcal{R}^{*}(\Sigma)$ of (gauge equivalence classes) of irreducible flat connections on $P$. After perturbation of the flatness equation $F_{A}=0$, if necessary, the moduli space $\mathcal{R}^{*}(\Sigma)$ is a compact oriented zero-dimensional manifold. Define the Casson invariant

$$
\lambda(\Sigma)=\frac{1}{2} \# \mathcal{R}^{*}(\Sigma),
$$

where $\# \mathcal{R}^{*}(\Sigma)$ stands for the signed count of the (finitely many) points in $\mathcal{R}^{*}(\Sigma)$. Making sense of this definition requires a lot of work, which goes mostly into orienting $\mathcal{R}^{*}(\Sigma)$ and choosing proper perturbations.

3.2. Orientation. We say that $A \in \mathcal{R}^{*}(\Sigma)$ is non-degenerate if $H^{1}(\Sigma$; ad $A)=$ 0 . This is equivalent to saying that, for a choice of Riemannian metric on $\Sigma$, the elliptic operator

$$
K_{A}=\left(\begin{array}{cc}
0 & d_{A}^{*} \\
d_{A} & -* d_{A}
\end{array}\right)
$$

acting on the space $\left(\Omega^{0} \oplus \Omega^{1}\right)(\Sigma$; ad $P)$ has zero kernel. If all points in $\mathcal{R}^{*}(\Sigma)$ are non-degenerate then there are only finitely many of them. The sign of $A \in \mathcal{R}^{*}(\Sigma)$ is defined as $(-1)^{\mu(A)}$ where $\mu(A)$ is the spectral flow of the family of operators $K_{A(t)}$ along a path $A(t)$ from the product connection $\theta$ to $A$. The quantity $\mu(A)$ reduced modulo 8 only depends on $A$ and not on a particular choice of $A(t)$, and is referred to as the Floer index of $A$. If $\mathcal{R}^{*}(\Sigma)$ fails to be non-degenerate, it is perturbed and then oriented using a similar procedure.

3.3. Perturbations. In the degenerate situation, the flatness equation $F_{A}=$ 0 is perturbed into $F_{A}=* \nabla h$, where $h$ is a function on the connections defined as follows, see Taubes 48] and Herald [25].

A collection of disjoint closed loops $\gamma_{k}$ embedded in $\Sigma$ will be called a link. Given a link of $n$ loops, consider $n$ smooth functions $f_{k}: S U(2) \rightarrow$ $\mathbb{R}$ invariant with respect to conjugation, and define $h(A)$ as the sum of $f_{k}\left(\operatorname{hol}_{A}\left(\gamma_{k}\right)\right)$. For analytical reasons, one uses a little more sophisticated definition of $h(A)$ obtained by averaging the above over the neighboring links. More precisely, thicken the link $\left\{\gamma_{k}\right\}$ into a collection of embeddings $\gamma_{k}: S^{1} \times D^{2} \rightarrow \Sigma$ with disjoint images, and define

$$
h(A)=\sum_{k=1}^{n} \int_{D^{2}} f_{k}\left(\operatorname{hol}_{A}\left(\gamma_{k}\left(S^{1} \times\{z\}\right)\right)\right) \eta(z) d^{2} z,
$$


where $\operatorname{hol}_{A}\left(\gamma_{k}\left(S^{1} \times\{z\}\right)\right)$ stands for holonomy of $A$ around the loop $\gamma_{k}\left(S^{1} \times\right.$ $\{z\}), z \in D^{2}$, and $\eta$ is any smooth rotationally symmetric bump function on $D^{2}$ with support away from the boundary of $D^{2}$ and with integral one.

The moduli space of irreducible solutions of the equation $F_{A}=* \nabla h$ is denoted by $\mathcal{R}_{h}^{*}(\Sigma)$; a point $A$ in this space is called non-degenerate if the kernel of the operator $K_{A}+\operatorname{Hess}_{A} h$ vanishes. It turns out that there exist the so called abundant links which have the property that, for any small generic functions $f_{k}$, the moduli space $\mathcal{R}_{h}^{*}(\Sigma)$ is non-degenerate. The nondegenerate space $\mathcal{R}_{h}^{*}(\Sigma)$ consists of finitely many points. We orient it using the spectral flow of the operators $K_{A(t)}+\operatorname{Hess}_{A(t)} h$ as above.

The class of perturbations described above is large enough for our current purposes. We expand this class to include loops with a common base point to deal with equivariant aspects of gauge theory in Sections 6.4 and 9.1 compare with Floer [16] and Herald [26].

3.4. Properties of the Casson invariant. The above construction ends up in an integer valued invariant $\lambda(\Sigma)$ which only depends on $\Sigma$ and not on the choices made in its definition. We will concentrate on one specific property of this invariant, namely, that $\lambda(\Sigma)=\rho(\Sigma)(\bmod 2)$. To verify this property, one traditionally goes back to the original definition of the Casson invariant using representations spaces. One proves a surgery formula for the Casson invariant in terms of the Alexander polynomial, and then shows that the modulo 2 reduction of this formula gives the surgery formula for the Rohlin invariant. A more direct proof using the gauge theoretic definition of the Casson invariant and avoiding the Alexander polynomial altogether will be given in Section 9.1

3.5. Triangulation of manifolds in dimension four. The existence of an invariant $\lambda(\Sigma)$ lifting the Rohlin invariant to the integers provides for a negative solution of the triangulation conjecture in dimension four. Here is a sketch of the argument.

Let $X$ be a closed topological 4-manifold with intersection form $E_{8}$ (such a manifold exists by Freedman's classification), and suppose that it is homeomorphic to a simplicial complex. Without loss of generality, we will assume that the links of all vertices in this complex but maybe one are homeomorphic to $S^{3}$. The link of the remaining vertex, say $v$, is then a homotopy sphere $\Sigma$. Since $\pi_{1} \Sigma$ is trivial, it has no irreducible $S U(2)$ representations and hence $\rho(\Sigma)=\lambda(\Sigma)=0(\bmod 2)$. On the other hand, removing an open neighborhood of $v$ makes $X$ into a piecewise linear manifold (which is the same as a smooth manifold in dimension four) with boundary $\Sigma$ and the intersection form $E_{8}$. Hence $\rho(\Sigma)=1(\bmod 2)$, and this contradiction shows that $X$ could not have been homeomorphic to a simplicial complex.

The only use for the Casson invariant in this application is that it shows that the Rohlin invariant of a homotopy sphere vanishes. This fact can also be deduced from the Poincaré conjecture. 


\section{The Furuta-Ohta invariant}

Both Casson and Rohlin invariant are generalized in this section to invariants of $\mathbb{Z}[\mathbb{Z}]$-homology $S^{1} \times S^{3}$, which are smooth 4-manifolds satisfying certain homological conditions. The equality $\lambda(\Sigma)=\rho(\Sigma)(\bmod 2)$ that we had in dimension three becomes a conjecture for these generalized invariants. We explain how this conjecture is related to the triangulation conjecture in dimensions five and higher.

4.1. Homology $S^{1} \times S^{3}$. A $\mathbb{Z}[\mathbb{Z}]-$ homology $S^{1} \times S^{3}$ is a smooth oriented 4manifold $X$ such that $H_{*}(X ; \mathbb{Z})=H_{*}\left(S^{1} \times S^{3} ; \mathbb{Z}\right)$ and $H_{*}(\tilde{X} ; \mathbb{Z})=H_{*}\left(S^{3} ; \mathbb{Z}\right)$, where $\tilde{X}$ is the universal abelian cover of $X$.

An ample source of examples of $\mathbb{Z}[\mathbb{Z}]$-homology $S^{1} \times S^{3}$ is provided by the following operation. Given a homology cobordism $W$ from a homology sphere $\Sigma$ to itself, form its closure $\bar{W}$ by identifying the two copies of $\Sigma$ in the boundary of $W$ by the identity map. The closure $\bar{W}$ is always a $\mathbb{Z}[\mathbb{Z}]$-homology $S^{1} \times S^{3}$. For instance, the closure of the product cobordism $[0,1] \times \Sigma$ is the product $S^{1} \times \Sigma$, and the closure of a mapping cylinder $W_{\tau}$ is the mapping torus $X_{\tau}=([0,1] \times \Sigma) /(0, x) \sim(1, \tau(x))$.

Another series of examples is generated by $S^{1}$-bundles $X \rightarrow Y$ over $3-$ manifolds $Y$ with $H_{*}(Y ; \mathbb{Z})=H_{*}\left(S^{1} \times S^{2} ; \mathbb{Z}\right)$. These examples were pointed out to us at the McMaster conference by Scott Baldridge, as they arise in his work on circle actions and Seiberg-Witten theory. An application of the Gysin exact sequence shows that, if the Euler class $e \in H^{2}(Y ; \mathbb{Z})$ of the $S^{1}$-bundle generates the group $H^{2}(Y ; \mathbb{Z})=\mathbb{Z}$, the manifold $X$ has integral homology of $S^{1} \times S^{3}$. However, the second condition that $H_{*}(\tilde{X} ; \mathbb{Z})=$ $H_{*}\left(S^{3} ; \mathbb{Z}\right)$ is only satisfied if $H_{*}(\tilde{Y} ; \mathbb{Z})=H_{*}\left(S^{2} ; \mathbb{Z}\right)$, which is equivalent to saying that the Alexander polynomial of $Y$ is trivial. It should be noted that there are plenty of manifolds $Y$ with homology of $S^{1} \times S^{2}$ whose Alexander polynomial is trivial; for instance, any $Y$ obtained by 0 -surgery on a knot in $S^{3}$ with trivial Alexander polynomial (such as untwisted Whitehead double) will do.

More examples come from 2-knots in the 4-sphere, surgery along which produces a manifold with the homology of $S^{1} \times S^{3}$. To satisfy the homological condition on the universal abelian cover of this manifold, one would start with a 2-knot with trivial Alexander polynomial. Such knots are readily constructed, for example as the $k$-fold twist-spin of a knot whose $k$-fold branched cover is a homology sphere.

4.2. Extension of the Rohlin invariant. Let $X$ be a $\mathbb{Z}[\mathbb{Z}]-$ homology $S^{1} \times S^{3}$ and choose an embedded 3-manifold $M \subset X$ whose fundamental class generates $H_{3}(X ; \mathbb{Z})=\mathbb{Z}$. Note that $M$ need not be a homology sphere. We define

$$
\rho(X)=\rho(M, \sigma) \quad(\bmod 2)
$$

where $\sigma$ is a spin structure on $M$ induced from $X$. This is a well defined invariant of $X$ independent of the choices made in its definition, compare 
with 35. For example, if $X=\bar{W}$ is the closure of a homology cobordism $W$ from a homology sphere $\Sigma$ to itself then $\rho(X)=\rho(\Sigma)(\bmod 2)$.

4.3. Definition of the Furuta-Ohta invariant. Let $X$ be a $\mathbb{Z}[\mathbb{Z}]-$ homology $S^{1} \times S^{3}$ and $\mathcal{M}^{*}(X)$ the moduli space of irreducible ASD connections on a trivial $S U(2)$ bundle $P \rightarrow X$. Note that all such connections are flat by Chern-Weil theory.

The formal dimension of $\mathcal{M}^{*}(X)$ is equal to $8 c_{2}(P)[X]-3\left(1-b_{1}+\right.$ $\left.b_{2}^{+}\right)(X)=0$. After perturbing the ASD equation $F_{A}^{+}=0$ if necessary, $\mathcal{M}^{*}(X)$ is a compact oriented zero dimensional manifold (it is worth mentioning that the perturbed ASD connections need no longer be flat or even perturbed flat). The Furuta-Ohta invariant of $X$ is then defined as

$$
\lambda_{F O}(X)=\frac{1}{4} \# \mathcal{M}^{*}(X),
$$

where $\# \mathcal{M}^{*}(X)$ stands for a signed count of points in $\mathcal{M}^{*}(X)$. The compactness of $\mathcal{M}^{*}(X)$ is guaranteed by the condition $H_{*}(\tilde{X} ; \mathbb{Z})=H_{*}\left(S^{3} ; \mathbb{Z}\right)$, see Furuta-Ohta [21. As with the Casson invariant, the main work goes into orienting $\mathcal{M}^{*}(X)$ and choosing proper perturbations.

4.4. Orientation. The moduli space $\mathcal{M}^{*}(X)$ is called non-degenerate if the ASD operator

$$
D_{A}=d_{A}^{*} \oplus d_{A}^{+}: \Omega^{1}(X ; \operatorname{ad} P) \rightarrow\left(\Omega^{0} \oplus \Omega_{+}^{2}\right)(X ; \operatorname{ad} P)
$$

has trivial cokernel for every $A \in \mathcal{M}^{*}(X)$. A non-degenerate $\mathcal{M}^{*}(X)$ is oriented using the following construction from the Donaldson theory. Let $\mathcal{B}(X)$ be the space of the gauge equivalence classes of connections on $P \rightarrow X$, and $\Lambda_{X}$ the determinant bundle of the family $D_{A}$ over $\mathcal{B}(X)$. This is a real line bundle with the property that, over $\mathcal{M}^{*}(X) \subset \mathcal{B}(X)$, it restricts to the orientation bundle of $\mathcal{M}^{*}(X)$. According to Donaldson, the bundle $\Lambda_{X}$ is trivial over $\mathcal{B}(X)$, and a choice of trivialization of $\Lambda_{X}$ given by an orientation of $H^{1}(X ; \mathbb{R})=\mathbb{R}$ (called homology orientation) fixes an orientation of $\mathcal{M}^{*}(X)$. If $\mathcal{M}^{*}(X)$ fails to be non-degenerate, it is perturbed first and then oriented using a similar construction.

4.5. Perturbations. To make sense of the above definition of $\lambda_{F O}(X)$, the ASD equation $F_{A}^{+}=0$ defining $\mathcal{M}^{*}(X)$ may need to be perturbed into $F_{A}^{+}=\sigma(A)$ using admissible perturbations $\sigma(A) \in \Omega_{+}^{2}(X$; ad $P)$. The latter are constructed as follows, compare with Donaldson [12].

Let us consider an embedding $\psi: S^{1} \rightarrow X$ and extend it to an embedding $\psi: S^{1} \times N^{3} \rightarrow X$ where $N^{3}$ is an oriented 3-manifold. For any connection $A$ in $P$ denote by $\operatorname{hol}_{A}\left(\psi\left(S^{1} \times\{x\}\right), s\right) \in S U(2)$ the holonomy of $A$ around the loop $\psi\left(S^{1} \times\{x\}\right)$ starting at the point $\psi(s, x)$. Let $\Pi: S U(2) \rightarrow \mathfrak{s u}(2)$ be the projection given by

$$
\Pi(u)=u-\frac{1}{2} \operatorname{tr}(u) \cdot \mathrm{Id} .
$$


Assigning $\Pi \operatorname{hol}_{A}\left(\psi\left(S^{1} \times\{x\}\right), s\right)$ to $\psi(s, x) \in X$ defines a section of ad $P$ over $\psi\left(S^{1} \times N^{3}\right)$. Now, given a form $\nu \in \Omega_{+}^{2}(X)$ supported in $\psi\left(S^{1} \times N^{3}\right)$, define a section

$$
\sigma(\nu, \psi, A) \in \Omega_{+}^{2}(X, \operatorname{ad} P)
$$

by taking tensor product of $\Pi \operatorname{hol}_{A}\left(\psi\left(S^{1} \times\{x\}\right), s\right)$ with $\nu$ over $\psi\left(S^{1} \times N^{3}\right)$ and letting it be zero otherwise.

More generally, consider a collection of embeddings $\psi_{k}: S^{1} \rightarrow X, k=$ $1, \ldots, n$, with disjoint images, called a link, and extend it to a collection of embeddings $\psi_{k}: S^{1} \times N_{k}^{3} \rightarrow X$ as above so that the $\psi_{k}\left(S^{1} \times N_{k}^{3}\right)$ are still disjoint. For any choice of $n$ smooth functions $\bar{f}_{1}, \ldots, \bar{f}_{n}:[-2,2] \rightarrow \mathbb{R}$ with vanishing derivatives at \pm 2 , define admissible perturbation

$$
\sigma(A)=\sum_{k=1}^{n} \partial \bar{f}_{k} \cdot \sigma\left(\nu_{k}, \psi_{k}, A\right),
$$

where $\partial \bar{f}_{k}$ is the function $\bar{f}_{k}^{\prime}$ evaluated at $\operatorname{tr} \operatorname{hol}_{A}\left(\psi_{k}\left(S^{1} \times\{x\}\right), s\right)$, and $\nu_{k}$ are real valued self-dual forms on $X$, each supported in its respective $\psi_{k}\left(S^{1} \times\right.$ $\left.N_{k}^{3}\right)$.

Given an admissible perturbation $\sigma$, the set of the gauge equivalence classes of irreducible solutions of the equation $F_{A}^{+}=\sigma(A)$ will be denoted by $\mathcal{M}_{\sigma}^{*}(X)$. There exist the so called abundant links which have the property that, for any small generic functions $\bar{f}_{k}$, the moduli space $\mathcal{M}_{\sigma}^{*}(X)$ is nondegenerate (the non-degeneracy condition here means that the cokernel of the perturbed ASD operator (4) vanishes). Once $\mathcal{M}_{\sigma}^{*}(X)$ is non-degenerate it is oriented as in Section 4.4

The class of admissible perturbations will be expanded in Section 10.7 to handle the equivariant gauge theory.

4.6. Properties of the Furuta-Ohta invariant. One can show that the above construction ends up in an invariant which only depends on $X$ and a choice of orientation of $H^{1}(X ; \mathbb{R})$. Note that this invariant can be viewed as one quarter of a degree zero Donaldson polynomial of $X$, except the latter is formally not defined for trivial bundles or manifolds with $b_{2}^{+}=0$.

Next we wish to discuss the factor of one quarter in the definition of the Furuta-Ohta invariant (3) vs. the factor of one half for the Casson invariant (11). The reason for the extra one half is a 2-fold symmetry arising from the action of $H^{1}\left(X ; \mathbb{Z}_{2}\right)=\mathbb{Z}_{2}$ on $\mathcal{M}^{*}(X)$. This action can be described as follows.

Let us view $\chi \in H^{1}\left(X ; \mathbb{Z}_{2}\right)$ as a homomorphism from $\pi_{1} X$ to $\mathbb{Z}_{2}=\{ \pm 1\}$. As such, it defines a flat complex line bundle $L_{\chi}$. Since $\chi$ lifts to an integral homology class, the bundle $L_{\chi}$ is trivial and hence the bundles $P$ and $P \otimes L_{\chi}$ are isomorphic. Then $\chi$ acts on $\mathcal{M}^{*}(X)$ by assigning to a connection $A$ in $P$ the connection $A \otimes \chi$ in $P \otimes L_{\chi}=P$ induced by $A$ and $\chi$. If one views $A$ as a representation $A: \pi_{1} X \rightarrow S U(2)$, the above action is given by the formula $\chi(A)(g)=\chi(g) A(g)$ for any $g \in \pi_{1} X$. 
Proposition 4.1. The above action of $H^{1}\left(X ; \mathbb{Z}_{2}\right)$ on $\mathcal{M}^{*}(X)$ is free.

Proof. Let us view $\mathcal{M}^{*}(X)$ as the irreducible part of the $S U(2)$ representation variety of $\pi_{1} X$. Suppose that $A: \pi_{1} X \rightarrow S U(2)$ is a fixed point of $\chi: \mathcal{M}^{*}(X) \rightarrow \mathcal{M}^{*}(X)$ then there exists a $u \in S U(2)$ such that $\chi(g) A(g)=u A(g) u^{-1}$ for all $g \in \pi_{1} X$. In particular, by applying $\chi$ twice, we see that $u^{2}$ must commute with the image of $A$ in $S U(2)$. Since $A$ is irreducible, this is only possible if $u^{2}= \pm 1$. The case of $u^{2}=1$ should be excluded because then $u= \pm 1$ and $-A(g)=A(g)$ at least for one $g \in \pi_{1} X$, which is impossible. Therefore, $u^{2}=-1$ and, up to conjugation, $u=i$. This means that $A$ is a binary dihedral representation, that is, its image is contained in $S_{i} \cup j \cdot S_{i}$, where $S_{i}$ is the circle of unit complex numbers in $S U(2)$.

The representation $A$ maps the subgroup $\pi_{1} \tilde{X}=\left[\pi_{1} X, \pi_{1} X\right]$ of $\pi_{1} X$ into the commutator subgroup of the binary dihedral group. The latter is the unit complex circle; in particular, it is abelian. Therefore, the restriction of $A$ onto $\pi_{1} \tilde{X}$ factors through $H_{1}(\tilde{X} ; \mathbb{Z})=0$ and hence $A$ itself factors through $H_{1}(X ; \mathbb{Z})=\pi_{1} X / \pi_{1} \tilde{X}=\mathbb{Z}$. This contradicts the irreducibility of $A$.

Since the action of $H^{1}\left(X ; \mathbb{Z}_{2}\right)$ on $\mathcal{M}^{*}(X)$ is free, there exists an equivariant admissible perturbation $\sigma$ such that $\mathcal{M}_{\sigma}^{*}(X)$ is non-degenerate and still admits a free action of $H^{1}\left(X ; \mathbb{Z}_{2}\right)$, compare with Section 10.7. This action is orientation preserving, see Donaldson 12, therefore, $\lambda_{F O}(X)$ is at worst a half-integer. In Section [5] we will interpret $\lambda_{F O}(\bar{W})$ as a Floer Lefschetz number and show that $\lambda_{F O}(\bar{W})$ is always an integer.

4.7. Applications. The main topological applications of the Furuta-Ohta invariant stem from the following two conjectures.

Conjecture 4.2. Let $X$ be a $\mathbb{Z}[\mathbb{Z}]$-homology $S^{1} \times S^{3}$ then $\lambda_{F O}(X)=\rho(X)$ $(\bmod 2)$.

Conjecture 4.3. Let $X$ be a $\mathbb{Z}[\mathbb{Z}]$-homology $S^{1} \times S^{3}$ which admits an orientation reversing diffeomorphism inducing an orientation preserving map on $H^{1}(X ; \mathbb{R})$. Then $\lambda_{F O}(X)=0$.

First, we show how these two conjectures would disprove the triangulation conjecture in dimensions $n \geq 5$. Recall from Section 2.6 that, in order to do that, it would be sufficient to show vanishing of the Rohlin invariant of any homology sphere $\Sigma$ having second order in $\Theta^{3}$. Given such a $\Sigma$, consider a homology cobordism $W$ from $-\Sigma$ to $\Sigma$ so that $\partial W=\Sigma \cup \Sigma$. Identify the boundary components of $W$ using the identity map. Let $X$ be an orientable double cover of the resulting non-orientable manifold, then $X$ is a homology $S^{1} \times S^{3}$ admitting an orientation reversing involution which induces an identity map on $H^{1}(X ; \mathbb{R})$. Conjecture 4.3 now implies that $\lambda_{F O}(X)=0$, and Conjecture 4.2 implies that $\rho(\Sigma)=\rho(X)=\lambda_{F O}(X)=0$ $(\bmod 2)$. 
That no two homology spheres with non-trivial Rohlin invariant can be homology cobordant to each other via a simply connected homology cobordism $W$ would follow by applying Conjecture 4.2 to the double of $W$.

4.8. Rohlin's invariant and the homotopy $S^{1} \times S^{3}$. Another application of Conjecture 4.2 is to the surgery-theoretic classification of smooth manifolds of the homotopy type of $S^{1} \times S^{3}$. In summary, if surgery theory 'worked' in dimension four as it does in higher dimensions, then there should exist a fake homotopy $S^{1} \times S^{3}$ with non-trivial Rohlin invariant. On the other hand, Conjecture 4.2 would mean that there is no such manifold. This would imply a failure of exactness of the smooth surgery sequence, namely that the $L$-group $L_{5}(\mathbb{Z}[\mathbb{Z}])$ does not act on the structure set of $S^{1} \times S^{3}$. Although the existence of non-diffeomorphic, $s$-cobordant 4-manifolds implies that the surgery sequence is not exact for simply connected 4-manifolds, we know of no example where the group $L_{5}(\mathbb{Z}[\pi])$ fails to act.

We will briefly review the surgery calculation, and refer the reader to Wall's book [52] and the excellent survey of Kirby and Taylor 28 for further details and references. We remind the reader that we are concerned here with smooth manifolds; the tools of surgery theory work better in the topological case, and the calculations are somewhat different. In particular, any homotopy $S^{1} \times S^{3}$ is homeomorphic to the real one. The fake smooth $S^{1} \times S^{3}$ predicted by the surgery sequence would be in a sense the simplest possible orientable manifold; the fake $\mathbf{R} P^{4}$ constructed by CappellShaneson [10] (see also [14]) is also detected by a codimension-one Rohlin invariant.

To understand this prediction, consider the (hypothetical) surgery sequence for the structure set of $Y=S^{1} \times S^{3}$,

$$
[\Sigma Y, G / P L] \stackrel{\theta}{\longrightarrow} L_{5}(\mathbb{Z}[\mathbb{Z}]) \stackrel{\gamma}{\longrightarrow} \mathcal{S}(Y) \stackrel{N}{\longrightarrow}[Y, G / P L] .
$$

The hypothetical part on which we concentrate is whether the map called $\gamma$ giving the action of $L_{5}(\mathbb{Z}[\mathbb{Z}])$ on $\mathcal{S}(Y)$ is actually defined. (Kirby and Taylor [28] explain that a stabilized (with respect to repeated connected sum with $S^{2} \times S^{2}$ ) version of $\gamma$ is defined and fits into an exact surgery sequence for a 'stable' structure set $\overline{\mathcal{S}}$ ). Since $L_{5}(\mathbb{Z})=0$, there are isomorphisms

$$
L_{5}(\mathbb{Z}[\mathbb{Z}]) \cong L_{5}(\mathbb{Z}) \oplus L_{4}(\mathbb{Z}) \stackrel{\sigma / 8}{\longrightarrow} \mathbb{Z}
$$

given by a codimension-one signature, see Shaneson [46]. On the other hand, the calculation

$$
[\Sigma Y, G / P L]=\left[S^{2} \vee S^{4} \vee S^{5}, G / P L\right] \cong \mathbb{Z}_{2} \oplus \mathbb{Z}
$$

and the fact that $\theta$ is a homomorphism, see Wall [52, reduce the calculation of $\theta$ to understanding the 4-dimensional surgery map

$$
\pi_{4}(G / P L) \rightarrow L_{4}(\mathbb{Z}) \stackrel{\sigma / 8}{\longrightarrow} \mathbb{Z} .
$$


But an element of $\pi_{4}(G / P L)$ is a normal map $V^{4} \rightarrow S^{4}$, so that the normal bundle of $V$ pulls back from a bundle over $S^{4}$. This implies that $V$ is in fact spin, so that Rohlin's theorem tells us that the image of $\theta$ has index 2 .

In other words, if there were a realization of the action of $L_{5}(\mathbb{Z}[\mathbb{Z}])$ on $\mathcal{S}(Y)$ (i.e. if the map $\gamma$ existed) then there would exist a nontrivial element in $\mathcal{S}(Y)$. It is easy to check that there is no self-homotopy equivalence of $Y$ realizing this element. Hence the conjectured equality of Furuta-Ohta and Rohlin invariants implies that the surgery sequence is not exact.

The existence of a fake $S^{1} \times S^{3}$ is of course related to the problem discussed in Section 2.7 of finding a simply connected homology cobordism between a Rohlin invariant-one homology sphere and itself. For gluing the boundary components of such a cobordism would give a homotopy $S^{1} \times S^{3}$ with nontrivial Rohlin invariant. The converse does not necessarily hold, because a homotopy $S^{1} \times S^{3}$ would not necessarily have a homology sphere carrying the third homology. Similarly, a homology sphere $\Sigma$ with $\rho(\Sigma)=1$ that is of order two in $\Theta^{3}$ (in the strong sense that $\Sigma \# \Sigma$ bounds a contractible manifold) would give rise to a fake non-orientable manifold homotopy equivalent to $S^{1} \widetilde{\times} S^{3}$. As in the orientable case, the smooth surgery sequence would predict the existence of such a manifold coming from the action of $L_{5}\left(\mathbb{Z}\left[\mathbb{Z}^{-}\right]\right)$. See Akbulut [1] for a stabilized version of this manifold.

\section{The Floer homology}

Floer [16] associated with every homology sphere $\Sigma$ eight abelian groups $I_{k}(\Sigma), 0 \leq k \leq 7$, called (instanton) Floer homology, which ramify the Casson invariant in that

$$
\lambda(\Sigma)=\frac{1}{2} \sum_{k}(-1)^{k} \operatorname{rk} I_{k}(\Sigma) .
$$

The definition is as follows. The Floer homology is the homology of the Floer chain complex $I C_{*}(\Sigma)$. The free abelian group $I C_{k}(\Sigma)$ is generated by the points $A \in \mathcal{R}^{*}(\Sigma)$ of Floer index $\mu(A)=k(\bmod 8)$, where the flat moduli space $\mathcal{R}^{*}(\Sigma)$ may need to be perturbed first, using admissible perturbations of Section 3.3 to make it non-degenerate. The differential

$$
\partial: I C_{k}(\Sigma) \rightarrow I C_{k-1}(\Sigma)
$$

is given by counting (perturbed) ASD connections over the cylinder $\mathbb{R} \times \Sigma$ with proper boundary conditions. The Floer homology is functorial with respect to cobordisms between homology spheres. We will be mostly interested in the case of a homology cobordism $W$ from a homology sphere $\Sigma_{0}$ to a homology sphere $\Sigma_{1}$. In this case, we have a well defined homomorphism $W_{*}: I_{*}\left(\Sigma_{0}\right) \rightarrow I_{*}\left(\Sigma_{1}\right)$ of degree zero obtained by counting ASD connections on a trivial $S U(2)$ bundle over $W$. Again, the ASD equation may need to be perturbed, in which case we use perturbations of the type described in Section 4.5, extended to match perturbations on $\partial W$. 
Let $W$ be a homology cobordism from a homology sphere $\Sigma$ to itself and $W_{k}: I_{k}(\Sigma) \rightarrow I_{k}(\Sigma)$ the automorphisms induced by $W$ in Floer homology. Define the (Floer) Lefschetz number of $W$ by the formula

$$
\text { Lef }(W)=\sum_{k=0}^{7}(-1)^{k} \operatorname{tr}\left(W_{k}\right) \text {. }
$$

Let $\bar{W}$ be the closure of $W$ then the usual gluing arguments can be used to show that

$$
\text { Lef }(W)=2 \lambda_{F O}(\bar{W}) .
$$

For example, the product cobordism $[0,1] \times \Sigma$ induces the identity map in Floer homology and hence Lef $([0,1] \times \Sigma)=2 \lambda(\Sigma)$; on the other hand, the closure of the product cobordism is $S^{1} \times \Sigma$, therefore, $\lambda_{F O}\left(S^{1} \times \Sigma\right)=\lambda(\Sigma)$. An extension of this calculation to mapping tori will be discussed in Section 6 .

Proposition 5.1. For any homology cobordism $W$ from a homology sphere $\Sigma$ to itself, the Lefschetz number Lef $(W)$ is even.

Proof. The homomorphism $W_{*}$ commutes with the $u$-map of Frøyshov [18]. The result now follows because the $u$-map provides isomorphisms (over the rationals) $I_{k}(\Sigma)=I_{k+4}(\Sigma)$ for all $k$.

Together with Proposition 4.1 this implies that the Furuta-Ohta invariant of the $\mathbb{Z}[\mathbb{Z}]$-homology $S^{1} \times S^{3}$ of the type $X=\bar{W}$ is always an integer. For manifolds of this type, Conjectures 4.2 and 4.3 can be reformulated as follows. The first conjecture asserts that $1 / 2 \operatorname{Lef}(W)=\rho(X)(\bmod 2)$ or, equivalently, that the modulo 2 reduction of $1 / 2$ Lef $(W)$ is independent of the choice of $W$. The second conjecture asserts that Lef $(W)=0$ if $W$ admits an orientation reversing diffeomorphism.

Since all $\mathbb{Z}[\mathbb{Z}]$-homology $S^{1} \times S^{3}$ that arise in applications described in Section 4.7 are of the type $X=\bar{W}$, proving the above two conjectures about Lef $(W)$ would suffice for those applications. However, it is far from clear that an arbitrary $\mathbb{Z}[\mathbb{Z}]$-homology $S^{1} \times S^{3}$ (or even a homotopy $S^{1} \times S^{3}$ as discussed in Section 4.8) can be built in this fashion hence the full strength Conjectures 4.2 and 4.3 may be needed for applications described in Section 4.8 .

\section{MAPPING TORI}

Let $\Sigma$ be a homology sphere and $\tau: \Sigma \rightarrow \Sigma$ an orientation preserving diffeomorphism. Recall that the mapping torus of $\tau$ is the smooth 4-manifold $X_{\tau}=([0,1] \times \Sigma) /(0, x) \sim(1, \tau(x))$ with the product orientation. In this section, we give explicit formulas for $\lambda_{F O}\left(X_{\tau}\right)$ in the case of finite order $\tau$; note that the theory has a rather different character depending on whether $\tau$ has fixed points or not. Our formulas first express $\lambda_{F O}\left(X_{\tau}\right)$ in terms of the equivariant Casson invariant, and then identify the latter as a linear 
combination of the (regular) Casson invariant and certain classical knot invariants. We use these explicit formulas to verify Conjectures 4.2 and 4.3 for the mapping tori of finite order diffeomorphisms.

6.1. The definition of the equivariant Casson invariant. Let $\tau: \Sigma \rightarrow$ $\Sigma$ be an orientation preserving diffeomorphism of finite order. It induces a map $\tau^{*}: \mathcal{R}^{*}(\Sigma) \rightarrow \mathcal{R}^{*}(\Sigma)$ via pull back of flat connections. Let $\mathcal{R}^{\tau}(\Sigma)$ be the fixed point set of $\tau^{*}$. After perturbing the flatness equation $F_{A}=0$, if necessary, $\mathcal{R}^{\tau}(\Sigma)$ is a compact canonically oriented manifold of dimension zero. We define the equivariant Casson invariant as

$$
\lambda^{\tau}(\Sigma)=\frac{1}{2} \# \mathcal{R}^{\tau}(\Sigma)
$$

where $\# \mathcal{R}^{\tau}(\Sigma)$ stands for the signed count of points in $\mathcal{R}^{\tau}(\Sigma)$.

As we see, the definition follows closely that of the (regular) Casson invariant; however, there are a few important differences. First, the nondegeneracy of $\mathcal{R}^{\tau}(\Sigma)$ at a point $A$ means vanishing of the equivariant cohomology group $H_{\tau}^{1}(\Sigma$; ad $A)$, which may be strictly smaller than the group $H^{1}(\Sigma ;$ ad $A)$. Therefore, we can use equivariant perturbations as in 11 to achieve non-degeneracy of $\mathcal{R}^{\tau}(\Sigma)$ without achieving non-degeneracy of the full moduli space $\mathcal{R}^{*}(\Sigma)$ (the latter should not be expected anyway because of the equivariant transversality problem). The equivariant perturbations in question are first constructed on the quotient manifold $\Sigma / \tau$ by applying the procedure of Section 3.3 to a link $\gamma_{k}$ in $\Sigma / \tau$, and then lifted to $\Sigma$.

Second, the space $\mathcal{R}^{\tau}(\Sigma)$ is oriented using the equivariant spectral flow, which is the spectral flow of the operators (2) restricted to the subspace of $\left(\Omega^{0} \oplus \Omega^{1}\right)(\Sigma$, ad $P)$ invariant with respect to the induced action of $\tau$. This spectral flow may well be different from the spectral flow used to orient $\mathcal{R}^{*}(\Sigma)$; in short, the natural inclusion $\mathcal{R}^{\tau}(\Sigma) \rightarrow \mathcal{R}^{*}(\Sigma)$ is not necessarily orientation preserving.

6.2. Furuta-Ohta vs. equivariant Casson. This section is dedicated to expressing the Furuta-Ohta invariant for mapping tori of finite order diffeomorphisms in terms of the equivariant Casson invariant. We give a brief outline of the proof here and refer the reader to our paper 37. for all the details.

Theorem 6.1. If $\tau: \Sigma \rightarrow \Sigma$ has finite order then $\lambda_{F O}\left(X_{\tau}\right)=\lambda^{\tau}(\Sigma)$.

Proof. First observe that all solutions of the equation $F_{A}^{+}=0$ on $X_{\tau}$ are flat by Chern-Weil theory. A flat connection over $X_{\tau}$ is pulled back to a flat connection over $\Sigma$ via an inclusion $i: \Sigma \rightarrow X_{\tau}$, and this pull back map defines a two-to-one map $\mathcal{M}^{*}\left(X_{\tau}\right) \rightarrow \mathcal{R}^{\tau}(\Sigma)$. The latter can be easily seen by interpreting flat connections as representations of respective fundamental groups. We have a splitting exact sequence

$$
0 \longrightarrow \pi_{1}(\Sigma) \longrightarrow \pi_{1}\left(X_{\tau}\right) \longrightarrow \mathbb{Z} \longrightarrow 0 .
$$


Let $t$ be a generator of $\mathbb{Z}$ then every irreducible representation $A: \pi_{1}\left(X_{\tau}\right) \rightarrow$ $S U(2)$ determines and is uniquely determined by the pair $(\alpha, u)$ where $u=$ $A(t)$ and $\alpha=i^{*} A: \pi_{1} \Sigma \rightarrow S U(2)$ is an irreducible representation such that $\tau^{*} \alpha=u \alpha u^{-1}$ (in particular, the conjugacy class of $\alpha$ belongs to $\mathcal{R}^{\tau}(\Sigma)$ ). Replacing $u$ by $-u$ in the above gives rise to a new representation $\pi_{1}\left(X_{\tau}\right) \rightarrow$ $S U(2)$, which in fact is simply the image of $A$ under the action of $H^{1}\left(X ; \mathbb{Z}_{2}\right)$ on $\mathcal{M}^{*}\left(X_{\tau}\right)$. This results in a two-to-one correspondence between $\mathcal{M}^{*}\left(X_{\tau}\right)$ and $\mathcal{R}^{\tau}(\Sigma)$. Moreover, a direct calculation with cohomology shows that $H^{1}\left(X_{\tau} ; \operatorname{ad} A\right)=H_{\tau}^{1}\left(\Sigma ; \operatorname{ad} i^{*} A\right)$.

Let us assume for the moment that $\mathcal{R}^{\tau}(\Sigma)$ is non-degenerate. Then so is $\mathcal{M}^{*}\left(X_{\tau}\right)$, and to prove the identity $\lambda_{F O}\left(X_{\tau}\right)=\lambda^{\tau}(\Sigma)$, we only need to show that the above identification of the moduli spaces is orientation preserving. This can be achieved by first interpreting the orientation on $\mathcal{M}^{*}\left(X_{\tau}\right)$ in terms of the orientation transport of a family of ASD operators $D_{A(t)}$, see Nicolaescu 33. Next, the orientation transport can be identified with the orientation given by the equivariant spectral flow along the path $i^{*} A(t)$ by expanding forms on $X_{\tau}$ into Fourier series in the direction of $S^{1}$ and comparing the spectra of operators $D_{A(t)}$ and $K_{i^{*} A(t)}$, compare with Atiyah, Patodi and Singer [5].

Another way to compare the orientations is by viewing $X_{\tau}$ as an (orbifold) $S^{1}$-bundle over the quotient manifold $\Sigma / \tau$. One can apply adiabatic limit techniques to this situation; after that the result will follow from the fact that the equivariant spectral flow on $\Sigma$ equals the (regular) spectral flow on the quotient $\Sigma / \tau$.

Finally, if $\mathcal{R}^{\tau}(\Sigma)$ fails to be non-degenerate, perturb the flatness equation $F_{A}=0$ into $F_{A}=* \nabla h$ where $h$ is lifted from a perturbation $h^{\prime}$ on $\Sigma / \tau$. According to [11, there are enough such perturbations to make $\mathcal{R}^{\tau}(\Sigma)$ nondegenerate. Next, perturb the ASD equation $F_{A}^{+}=0$ into $F_{A}^{+}=\sigma(A)$ where $\sigma(A)$ is the self-dual part of the 2 -form $* \nabla h^{\prime}$ pulled back to $X_{\tau}$ via the projection $X_{\tau} \rightarrow \Sigma / \tau$. Note that $\sigma(A)$ is of the type described in Section 4.5 with $N_{k}^{3}=S^{1} \times D^{2}$. One can verify that there still exists the twoto-one correspondence $\mathcal{M}_{\sigma}^{*}\left(X_{\tau}\right) \rightarrow \mathcal{R}_{h}^{\tau}(\Sigma)$ between the perturbed moduli spaces, and that the perturbations $\sigma$ as described above are sufficient to make $\mathcal{M}_{\sigma}^{*}\left(X_{\tau}\right)$ non-degenerate. The latter essentially follows by comparing the abundancy concepts for the two types of perturbations and using the equality $H^{1}\left(X_{\tau} ; \operatorname{ad} A\right)=H_{\tau}^{1}\left(\Sigma ; \operatorname{ad} i^{*} A\right)$ of the respective Zariski tangent spaces.

6.3. Equivariant Casson: non-free actions. Let $\tau: \Sigma \rightarrow \Sigma$ be an orientation preserving diffeomorphism of finite order $n$, and suppose that the fixed point set of $\tau$ is non-empty. Then the quotient manifold $\Sigma^{\prime}=\Sigma / \tau$ is a homology sphere, and the projection $\Sigma \rightarrow \Sigma^{\prime}$ is a branched covering with branch set a knot $k \subset \Sigma^{\prime}$. The following is proved in Collin-Saveliev [11]. 
Theorem 6.2. In the situation described above, the equivariant Casson invariant is given by the formula

$$
\lambda^{\tau}(\Sigma)=n \cdot \lambda\left(\Sigma^{\prime}\right)+\frac{1}{8} \sum_{m=0}^{n-1} \operatorname{sign}^{m / n}(k),
$$

where $\operatorname{sign}^{a}(k)$ is the Tristram-Levine equivariant knot signature, defined as the signature of the Hermitian form $\left(1-e^{2 \pi i a}\right) S+\left(1-e^{-2 \pi i a}\right) S^{t}$, for any choice of Seifert matrix $S$ of $k$.

Proof. The proof proceeds by pushing equivariant flat connections from $\Sigma$ down to singular connections over the quotient $\Sigma^{\prime}$ and using Herald's theorem 24] which asserts that an appropriate count of the latter connections is a certain linear combination of $\lambda\left(\Sigma^{\prime}\right)$ and equivariant knot signatures.

Corollary 6.3. Let $\tau: \Sigma \rightarrow \Sigma$ be a finite order orientation preserving diffeomorphism having fixed points then $\lambda_{F O}\left(X_{\tau}\right)=\rho\left(X_{\tau}\right)(\bmod 2)$.

Proof. This is equivalent to showing that $\lambda^{\tau}(\Sigma)=\rho(\Sigma)(\bmod 2)$ which follows from Theorem 6.2 by standard techniques of geometric topology, see for instance Viro [50].

6.4. Equivariant Casson: free actions. In case $\tau$ acts freely on $\Sigma$, the quotient $\Sigma^{\prime}=\Sigma / \tau$ is a homology lens space. It is easy to see that $\Sigma^{\prime}$ can be obtained by $(n / q)$-surgery on a knot $k$ in a homology sphere $Y$ where $n$ is the order of $\tau$ and $q$ is relatively prime to $n$. The following is proved in our paper [37.

Theorem 6.4. In the situation described above, the equivariant Casson invariant is given by the formula

$$
\lambda^{\tau}(\Sigma)=n \cdot \lambda(Y)+\frac{1}{8} \sum_{m=0}^{n-1} \operatorname{sign}^{m / n}(k)+\frac{q}{2} \Delta_{k}^{\prime \prime}(1),
$$

where $\Delta_{k}(t)$ is the Alexander polynomial of $k \subset Y$ normalized so that $\Delta_{k}(1)=1$ and $\Delta_{k}(t)=\Delta_{k}\left(t^{-1}\right)$.

Proof. The proof proceeds by pushing equivariant flat connections from $\Sigma$ to the homology lens space $\Sigma^{\prime}$ and identifying the count of the latter connections with a sum of invariants of the type discussed by Boyer-Nicas [7] and Boyer-Lines [6. Note that these invariants are different from Walker's invariant [51, in that they only count irreducible flat connections over $\Sigma^{\prime}$; their gauge theoretic definition is implicit in work of Cappell, Lee and Miller 9]. An application of surgery formulas and Herald's theorem on equivariant knot signatures [24] completes the proof.

Let $Y_{n}$ be the $n$-fold cyclic cover of $Y$ branched along $k$. Observe that $Y_{n}$ is a homology sphere and that $\Sigma$ is obtained by $(1 / q)$-surgery on $Y_{n}$ along a lift $k_{n}$ of $k$. Combining Theorem 6.4 with Theorem 6.2 we see that

$$
\lambda^{\tau}(\Sigma)=\lambda^{\tau}\left(Y_{n}\right)+\frac{q}{2} \Delta_{k}^{\prime \prime}(1)
$$


Since we already know that $\lambda^{\tau}\left(Y_{n}\right)=\rho\left(Y_{n}\right)(\bmod 2)$, see Corollary 6.3, we will be able to conclude that $\lambda^{\tau}(\Sigma)=\rho(\Sigma)(\bmod 2)$ once we verify the following result, see 37.

Lemma 6.5. Let $Y$ be an integral homology sphere and $\pi: Y_{n} \rightarrow Y$ its $n$-fold cyclic branched covering with branch set a knot $k$. Let $k_{n}$ be the knot $\pi^{-1}(k)$ in $Y_{n}$. If $Y_{n}$ is an integral homology sphere then $\operatorname{arf}\left(k_{n}\right)=\operatorname{arf}(k)$ $(\bmod 2)$.

Corollary 6.6. Let $\tau: \Sigma \rightarrow \Sigma$ be a free finite order orientation preserving diffeomorphism then $\lambda_{F O}\left(X_{\tau}\right)=\rho\left(X_{\tau}\right)(\bmod 2)$.

6.5. Orientation reversal. Corollaries 6.3 and 6.6 verify Conjecture 4.2 for the mapping tori $X_{\tau}$ of finite order diffeomorphisms $\tau: \Sigma \rightarrow \Sigma$. To verify Conjecture 4.3 for such mapping tori, observe that any orientation reversing diffeomorphism $f: X_{\tau} \rightarrow X_{\tau}$ which preserves homology orientation can be viewed as a diffeomorphism $f:-X_{\tau} \rightarrow X_{\tau}$ preserving both orientation and homology orientation, so that $\lambda_{F O}\left(-X_{\tau}\right)=\lambda_{F O}\left(X_{\tau}\right)$. On the other hand, since $-X_{\tau}$ is the mapping torus of the orientation preserving diffeomorphism $\tau:-\Sigma \rightarrow-\Sigma$, we conclude that $\lambda_{F O}\left(-X_{\tau}\right)=\lambda^{\tau}(-\Sigma)$ by Theorem 6.1. The equivariant Casson invariant changes sign with the change of orientation, therefore, $\lambda_{F O}\left(-X_{\tau}\right)=-\lambda^{\tau}(\Sigma)=-\lambda_{F O}\left(X_{\tau}\right)$ and thus $\lambda_{F O}\left(X_{\tau}\right)$ must vanish.

\section{EXAMPLES}

In this section, we give examples of finite order maps $\tau: \Sigma \rightarrow \Sigma$ for which the Furuta-Ohta invariant and the Floer Lefschetz number can be computed explicitly using methods described above. An interesting observation is that the map $\left(W_{\tau}\right)_{*}: I_{*}(\Sigma) \rightarrow I_{*}(\Sigma)$ induced by the mapping cylinder $W_{\tau}$ of $\tau$ need not be identity even when $\tau$ acts trivially on the representation variety $\mathcal{R}^{*}(\Sigma)$. This has to do with the fact that $\left(W_{\tau}\right)_{*}$ is defined using a count of ASD connections over $W_{\tau}$ with signs which are not determined solely by the fundamental group.

7.1. Akbulut cork. By Akbulut cork we mean the smooth contractible 4-manifold $W$ obtained by attaching a two-handle to $S^{1} \times D^{3}$ along its boundary as shown in Figure 1. It can be embedded into a blown up elliptic surface $E(n) \#\left(-\mathbb{C} P^{2}\right)$ in such a way that cutting it out and re-gluing by an involution on $\Sigma=\partial W$ changes the smooth structure on $E(n) \#\left(-\mathbb{C} P^{2}\right)$ but preserves its homeomorphism type, see Akbulut [2] and Gompf and Stipsicz 23 .

The involution $\tau: \Sigma \rightarrow \Sigma$ simply interchanges the two link components, $k_{1}$ and $k_{2}$; this is best seen when the link is drawn in a symmetric form as in Figure 2

The manifold $\Sigma^{\prime}=\Sigma / \tau$ is obtained from $S^{3}$ by surgery on the knot $k^{*}$ which is the image of the link $k_{1} \cup k_{2}$, see Figure 3. Note that the canonical longitudes of $k_{1}$ and $k_{2}$ project onto a longitude of $k^{*}$ whose linking number 


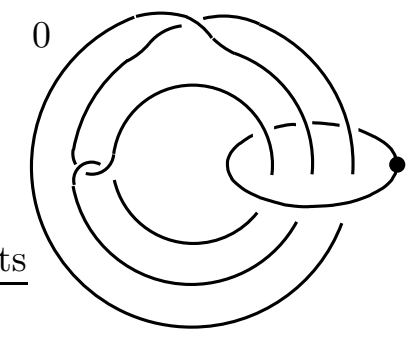

Figure 1.

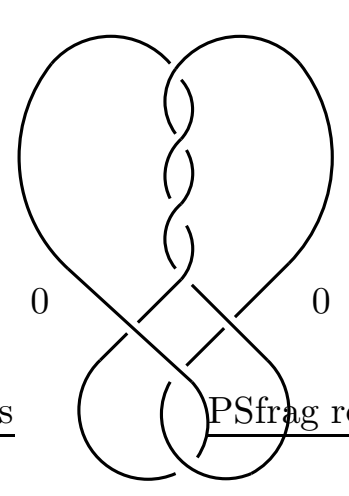

FiguRE 2.

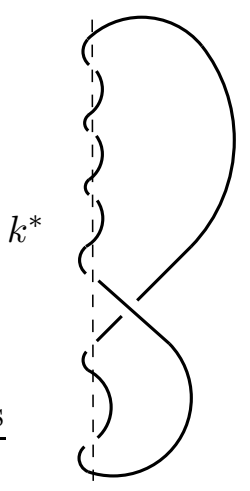

Figure 3.

with $k^{*}$ equals one. The dotted line in Figure 3 represents the branch set $k$ of $\Sigma \rightarrow \Sigma^{\prime}$. This picture can be viewed as a surgery description of the knot $k$ in $\Sigma^{\prime}=S^{3}$. A little exercise in Kirby calculus shows that $k$ can be obtained from the left-handed $(5,6)$-torus knot on six strings by adding one full left-handed twist on two adjacent strings. The signature of $k$ can differ by at most two from the signature of the left-handed $(5,6)$-torus knot, which equals 16 . Since sign $k$ must be divisible by eight, we conclude that $\operatorname{sign} k=16$. Therefore, $\lambda_{F O}\left(X_{\tau}\right)=\lambda^{\tau}(\Sigma)=2$ by Theorem 6.2 and Lef $\left(W_{\tau}\right)=2 \cdot \lambda_{F O}\left(X_{\tau}\right)=4$, see (15).

In fact, the latter formula provides enough information to describe the $\operatorname{map}\left(W_{\tau}\right)_{*}: I_{*}(\Sigma) \rightarrow I_{*}(\Sigma)$. According to [44, the Floer homology of $\Sigma$ is trivial in even degrees, and is a copy of $\mathbb{Z}$ in each of the odd degrees. Therefore, $\tau_{*}: I_{k}(\Sigma) \rightarrow I_{k}(\Sigma)$ is necessarily plus or minus identity for each $k$. Since the Lefschetz number of $\tau_{*}$ equals 4 , this implies that $\left(W_{\tau}\right)_{*}$ is minus identity.

It is worth mentioning that the character variety of $\Sigma$ is non-degenerate, and that the action induced by $\tau$ on $\mathcal{R}^{*}(\Sigma)$ is a non-trivial permutation, see [44.

7.2. Seifert fibered homology spheres. Let $\Sigma\left(a_{1}, \ldots, a_{n}\right)$ be a Seifert fibered homology sphere viewed as a link of singularity, and $\tau$ the involution 
on it induced by complex conjugation. It makes $\Sigma\left(a_{1}, \ldots, a_{n}\right)$ into a double branched cover of $S^{3}$ with branch set a Montesinos knot $k$. A straightforward application of Theorem6.2 shows that both $\lambda_{F O}\left(X_{\tau}\right)$ and $(1 / 2)$ Lef $\left(W_{\tau}\right)$ are equal to $1 / 8 \operatorname{sign} k$, which is also known as the $\bar{\mu}$-invariant of $\Sigma\left(a_{1}, \ldots, a_{n}\right)$, see Neumann 32 and Siebenmann [47. As a side note, we mention that the homology cobordism invariance of the $\bar{\mu}$-invariant, shown with the help of the orbifold Seiberg-Witten theory, is the main tool in proving Theorem 2.1 .

As a rule, the $\bar{\mu}$-invariant of $\Sigma\left(a_{1}, \ldots, a_{n}\right)$ differs from its Casson invariant, which indicates that the action $\tau_{*}: I_{*}\left(\Sigma\left(a_{1}, \ldots, a_{n}\right)\right) \rightarrow I_{*}\left(\Sigma\left(a_{1}, \ldots, a_{n}\right)\right)$ is non-trivial. With an extra effort, this action can be described explicitly. According to Fintushel-Stern [15], the group $I_{k}\left(\Sigma\left(a_{1}, \ldots, a_{n}\right)\right)$ is trivial if $k$ is even, and is a free abelian group of rank, say, $b_{k}$ if $k$ is odd. The following is proved in [40].

Theorem 7.1. The map $\tau_{*}: I_{k}\left(\Sigma\left(a_{1}, \ldots, a_{n}\right)\right) \rightarrow I_{k}\left(\Sigma\left(a_{1}, \ldots, a_{n}\right)\right)$ is identity if $k=1(\bmod 4)$ and minus identity if $k=-1(\bmod 4)$.

The character variety of any Seifert fibered homology sphere with (no more than) three singular fibers is non-degenerate, and one can show that the action induced on it by $\tau$ is trivial, see [40. The same is true for any number of singular fibers after one perturbs the character variety using equivariant perturbations as in [45].

\section{Circle bundles}

In this section we describe another class of $\mathbb{Z}[\mathbb{Z}]$-homology $S^{1} \times S^{3}$ for which both Conjectures 4.2 and 4.3 have been verified; it turns out that in all of these examples, both invariants $\lambda_{F O}$ and $\rho$ vanish. As mentioned in Section 4.1] these examples were pointed out to us by Scott Baldridge.

8.1. The manifolds. Let $Y$ be a closed oriented 3-manifold having integral homology of $S^{1} \times S^{2}$, and denote by $\Delta(t)$ its Alexander polynomial normalized so that $\Delta(1)=1$ and $\Delta\left(t^{-1}\right)=\Delta(t)$. Every manifold $Y$ as above can be obtained by 0 -surgery on a knot $k$ in a homology sphere $\Sigma$; the Alexander polynomial of $Y$ then equals the Alexander polynomial of $k \subset \Sigma$.

Let $\pi: X \rightarrow Y$ be an $S^{1}$-bundle classified by its Euler class $e \in H^{2}(Y ; \mathbb{Z})=$ $\mathbb{Z}$ and assume that $e=1$. Then one can use the Gysin exact sequence to show that $X$ has integral homology of $S^{1} \times S^{3}$. To ensure that $X$ is a $\mathbb{Z}[\mathbb{Z}]_{-}$ homology $S^{1} \times S^{3}$, the universal abelian cover $\tilde{X}$ should have homology of $S^{3}$; this is equivalent to having $\Delta(t)=1$. These are the manifolds $X$ that we will study in this section.

8.2. Calculating $\rho(X)$. Recall that $\rho(X)=\rho(M, \sigma)$ where $M \subset X$ is an embedded 3 -manifold representing a generator in $H_{3}(X ; \mathbb{Z})$ and $\sigma$ is the induced spin structure. A calculation with Gysin exact sequence shows that $M$ can be obtained by taking a surface $S$ carrying $H_{2}(Y ; \mathbb{Z})$ and then taking its preimage in $X$, which is of course a circle bundle over $S$. The spin 
structure on $X$ can be described as follows. There is an exact sequence of bundles

$$
0 \longrightarrow V \longrightarrow T X \longrightarrow \pi^{*} T Y \longrightarrow 0
$$

where $V$ is the vertical tangent space. This induces a bijection between spin structures on $Y$ and those on $X$. Thus the induced spin structure $\sigma$ on $M$ is given by taking a spin structure on $Y$ (either one will do) and restricting to $S$, and then using the same exact sequence to get a spin structure on $M$.

To calculate the Rohlin invariant $\rho(M, \sigma)$, consider the disk bundle $W \rightarrow$ $S$ with Euler class 1, whose boundary is $M$. The manifold $W$ is not spin but we still obtain

$$
\rho(M, \sigma)=(\operatorname{sign} W-S \cdot S) / 8+\operatorname{arf}(S)=\operatorname{arf}(S) \quad(\bmod 2),
$$

where $\operatorname{arf}(S)$ is the Arf-invariant of the induced spin structure. Since the Alexander polynomial of $Y$ vanishes, we conclude that $\operatorname{arf}(S)=0$.

8.3. Calculating $\lambda_{F O}(X)$. In this section, we find it convenient to work with $S O(3)$ flat connections rather than with $S U(2)$ ones. The two settings are equivalent due to the presence of a free orientation preserving $H^{1}\left(X ; \mathbb{Z}_{2}\right)$ action on $\mathcal{M}^{*}(X)$ whose quotient is the $S O(3)$ flat moduli space $\mathcal{M}^{*}(X, S O(3))$. Note that this remains true after a small generic equivariant perturbation $\sigma$ making $\mathcal{M}_{\sigma}^{*}(X)$ non-degenerate, see Proposition 4.1 and Section 10.7

Without loss of generality we will assume that $\pi_{2}(Y)=0$. The homotopy exact sequence of the $S^{1}$-bundle $\pi: X \rightarrow Y$ then implies that $\pi_{1} X$ is a central extension of $\pi_{1} Y$ by the integers,

$$
1 \longrightarrow \mathbb{Z} \longrightarrow \pi_{1} X \stackrel{\pi_{*}}{\longrightarrow} \pi_{1} Y \longrightarrow 1 \text {. }
$$

Let $h$ be a generator in $\mathbb{Z}$ then every irreducible representation $A: \pi_{1} X \rightarrow$ $S O(3)$ has the property that $A(h)=1$. Therefore, we have a natural identification $\pi^{*}: \mathcal{R}^{*}(Y, S O(3)) \rightarrow \mathcal{M}^{*}(X, S O(3))$ where $\mathcal{R}^{*}(Y, S O(3))$ is the $S O(3)$ character variety of $\pi_{1} Y$.

This identification induces an isomorphism $\pi^{*}: H^{1}(Y ; \operatorname{ad} A) \rightarrow H^{1}\left(X ; \operatorname{ad} \pi^{*} A\right)$ of Zariski tangent spaces, which is easily seen from the Gysin exact sequence

$0 \longrightarrow H^{1}(Y ; \operatorname{ad} A) \stackrel{\pi^{*}}{\longrightarrow} H^{1}\left(X ; \operatorname{ad} \pi^{*} A\right) \longrightarrow H^{0}(Y ; \operatorname{ad} A) \longrightarrow 0$

after one notes that $H^{0}(Y$; ad $A)$ vanishes because $A$ is irreducible. Thus the moduli space $\mathcal{M}^{*}(X, S O(3))$ is non-degenerate if and only if $\mathcal{R}^{*}(Y, S O(3))$ is. Should the non-degeneracy fail, both $\mathcal{R}^{*}(Y, S O(3))$ and $\mathcal{M}^{*}(X, S O(3))$ need to be perturbed. This can be done in a consistent manner as in the proof of Theorem 6.1 so that the pull back of connections via $\pi: X \rightarrow Y$ still provides a bijective correspondence between the perturbed moduli spaces. To show that this correspondence is orientation preserving, one can use adiabatic limit techniques, see Nicolaescu 34 .

Thus $\lambda_{F O}(X)$ equals one half times the signed count of points in the (perturbed) moduli space $\mathcal{R}^{*}(Y, S O(3))$. In computing the latter, we will 
rely on the papers 24] and 37], to which we refer the reader for all the details.

View $Y$ as the result of 0 -surgery on a knot $k$ in a homology sphere $\Sigma$. Let $Z=\Sigma \backslash N(k)$ be the knot $k$ exterior, and $m$ and $\ell$ be the canonical meridian and longitude on the torus $\partial Z$. Then the dual torus $\mathcal{P}=\mathcal{R}(\partial Z, U(1))$ has coordinates $(\varphi, \psi)$ such that the holonomies along $m$ and $\ell$ are equal to $\exp (i \varphi)$ and $\exp (i \psi)$, respectively. The inclusion $\partial Z \rightarrow Z$ induces a natural restriction map from a double cover of $\mathcal{R}(Z, S U(2))$ to $\mathcal{P}$ whose image, $\tilde{\mathcal{C}}$, is generically an immersed curve. Moreover, since $\Delta_{k}(t)=1$, this image consists of finitely many circles.

Let us consider the splitting $\mathcal{R}^{*}(Y, S O(3))=\mathcal{R}_{0}^{*}(Y, S O(3)) \cup \mathcal{R}_{1}^{*}(Y, S O(3))$, where $\mathcal{R}_{w}^{*}(Y, S O(3))$ stands for the moduli space of irreducible flat connections on an $S O(3)$ bundle $P$ having $w_{2}(P)=w \in H^{2}\left(Y ; \mathbb{Z}_{2}\right)=\mathbb{Z}_{2}$. Up to an overall constant, the signed count of points in $\mathcal{R}_{1}^{*}(Y, S O(3))$ equals the intersection number of $\tilde{\mathcal{C}}$ with the circle $\psi=\pi$ and hence equals $\Delta_{k}^{\prime \prime}(1)=0$. Similarly, the signed count of points in $\mathcal{R}_{0}^{*}(Y, S O(3))$ equals, up to an overall constant, the intersection number of $\tilde{\mathcal{C}}$ with the circle $\psi=0$. Since the circles $\psi=0$ and $\psi=\pi$ are homologous in $\mathcal{P}$, this intersection number is again equal to $\Delta_{k}^{\prime \prime}(1)=0$.

\section{A general approach to the conjectures}

To prove Conjecture 4.2, we would like to carry out a surgery program similar to the program showing the equality $\lambda(\Sigma)=\rho(\Sigma)(\bmod 2)$ in three dimensions. Many essential features of that proof, like reliance on Heegaard splittings and the Alexander polynomial, are not easily transferable one dimension higher. Therefore, our first step is giving a purely gauge theoretic proof of $\lambda(\Sigma)=\rho(\Sigma)(\bmod 2)$, see [36]. This proof is briefly described in Section 9.1. After that, we outline a surgery program in dimension four for proving Conjecture 4.2. The final step of this program, the calculation of the degree zero Donaldson polynomial for homology tori, is now complete (modulo some technical issues), see Section [10. while intermediate steps require a lot of work.

Verifying Conjecture 4.3 amounts to comparing the Furuta-Ohta invariants for a $\mathbb{Z}[\mathbb{Z}]$-homology $S^{1} \times S^{3}$ with two opposite orientations. We only know how to prove this conjecture in the situation when the ASD moduli space $\mathcal{M}^{*}(X)$ is non-degenerate; the general case is still out of our reach.

9.1. Homology 3-tori and the Casson invariant. The Casson invariant has the property that $\lambda(\Sigma)=\rho(\Sigma)(\bmod 2)$ for any homology sphere $\Sigma$, see Section 3.4. The traditional proof of this property involves several steps.

The first step takes us back to the original definition of the Casson invariant in terms of Heegaard splittings. This step relies on Taubes' theorem 48.

In the second step, surgery formulas are used to express the Rohlin and the Casson invariants in terms of their first, second and third difference 
quotients, see 3] or 43. The third difference quotients, $\rho^{\prime \prime \prime}(\Sigma)$ and $\lambda^{\prime \prime \prime}(Y)$, are invariants of a manifold $Y$ having integral homology of the 3 -torus (or a homology 3-torus, for short). Since the surgery formula for the Rohlin invariant happens to be the modulo two reduction of the surgery formula for the Casson invariant, showing that $\lambda(\Sigma)=\rho(\Sigma)(\bmod 2)$ amounts to verifying that $\lambda^{\prime \prime \prime}(Y)=\rho^{\prime \prime \prime}(Y)(\bmod 2)$.

According to Kaplan [27], the invariant $\rho^{\prime \prime \prime}(Y)$ equals the determinant of $Y$ defined as $\operatorname{det} Y=\left(a_{1} \cup a_{2} \cup a_{3}\right)[Y](\bmod 2)$, where $a_{1}, a_{2}, a_{3}$ is a basis in $H^{1}\left(Y ; \mathbb{Z}_{2}\right)$. The final step is then proving that $\lambda^{\prime \prime \prime}(Y)=\operatorname{det} Y(\bmod 2)$. This is accomplished by using the interpretation of Casson's difference quotients in terms of the Alexander polynomials of links.

Our approach eliminates the first step of the above program and hence the need for Taubes' theorem. Instead, we directly apply the Casson surgery formula, which in gauge theoretic terms is an easy corollary of the Floer exact triangle, see [17] and [8]. This expresses $\lambda(\Sigma)$ in terms of third difference quotients $\lambda^{\prime \prime \prime}(Y)$ where $\lambda^{\prime \prime \prime}(Y)$ now equals a proper count of flat connections on an $S O(3)$ bundle over $Y$ with a non-trivial second Stiefel-Whitney class $w$ (the invariant $\lambda^{\prime \prime \prime}(Y)$ does not depend on the choice of $w \neq 0$ ).

The final step of the proof, that is, showing that $\lambda^{\prime \prime \prime}(Y)=\operatorname{det} Y(\bmod 2)$, is done gauge-theoretically along the lines of our proof of Theorem 10.2. We refer the reader to [36] for all the details.

9.2. Round surgery. We would like to carry out a similar program in dimension four. The idea is to follow the above proof step by step in a category of 4 -manifolds $X$ over $S^{1}$, and reduce Conjecture 4.2 to the result about $\mathbb{Z}[\mathbb{Z}]$-homology 4 -tori proved in Theorem 10.2

More precisely, a 4 -manifold over $S^{1}$ is a smooth closed oriented manifold $X$ of dimension four with a preferred cohomology class $\alpha \in H^{1}(X ; \mathbb{Z})$. There are obvious notions of cobordism and surgeries of manifolds over $S^{1}$. Roughly speaking, we want to think of our 4-manifolds as 'looking like' they are the product of $S^{1}$ with a 3 -manifold, and thus the surgeries allowed should look like they are really given by $S^{1}$ times a surgery on the 3 -manifold. This brings into our discussion the concept of round surgery defined by Asimov 4. A four-dimensional round handle of index $k$ is a pair

$$
S^{1} \times\left(D^{k} \times D^{3-k}, S^{k-1} \times D^{3-k}\right)
$$

attached to a manifold with boundary. There are obvious notions of round handle decompositions, round surgeries etc. Asimov 4 showed that the existence (in dimensions at least four) of round handle decompositions is governed by the Euler characteristic. From this it is not difficult to prove the following result.

Proposition 9.1. Let $X$ be a smooth $\mathbb{Z}[\mathbb{Z}]$-homology $S^{1} \times S^{3}$. Then $X$ may be obtained from $S^{1} \times S^{3}$ via a series of round surgeries of indices 2 and 3 .

Unfortunately, the intermediate stages in the resulting cobordism might not have well defined Rohlin invariants (and their gauge theory invariants 
are not so good either). The problem would be rectified if we could make the intermediate stages look like $S^{1}$ times a 3 -manifold. To accomplish this, we need to restrict the kind of surgeries we allow. The restriction is in terms of the degree of the round surgery, which is by definition the integer $\left\langle\alpha, S^{1}\right\rangle$. If the degree of a round surgery is \pm 1 , then we can define appropriate gauge theoretic and Rohlin invariants, and then try to compare them. Thus we need to strengthen Proposition 9.1 to say that $X$ can be obtained by a series of round surgeries, each of which has degree \pm 1 .

This strengthening, although it sounds fairly innocent, seems to be very difficult to accomplish, and indeed there may need to be some modification in the program which we now describe. Assuming that there is some strengthened proposition along these lines, here are some ideas on how we might obtain a proof of Conjecture 4.2. Except for the very last one, each of the steps in this outline still has many details to be filled in.

The main point is that there seem to exist surgery formulas for both Rohlin and Furuta-Ohta invariants for round surgeries of degree \pm 1 . These have a form that one might expect by thinking about surgery on $S^{1}$ times a 3-manifold. Namely, different framings for a surgery on $X$ give rise to manifolds $X_{1}$ and $X_{0}$, where $X_{1}$ has the same homology as $X$, and $b_{1}\left(X_{0}\right)=$ $b_{1}(X)+1$. (The notations are supposed to suggest +1 and 0 surgery on a 3-manifold, respectively). Both the Rohlin and the Furuta-Ohta invariants of $X$ can be written as the sum of invariants for $X_{0}$ and $X_{1}$. As in the 3-dimensional theory, we need to have a direct verification that the two invariants coincide for manifolds with sufficiently large $b_{1}$; it turns out that $b_{1}=4$ is as far as we need to go. We have actually carried out this final step; in the next section we define the Rohlin and Furuta-Ohta invariants for homology 4-tori and outline our theorem that they in fact coincide.

\section{Gauge theory on homology tori}

We study the degree zero Donaldson polynomial for $\mathbb{Z}[\mathbb{Z}]$-homology 4-tori and relate it to a properly defined Rohlin invariant. A similar result holds for homology 3-tori, which leads to a purely gauge theoretic proof of the fact that the Casson invariant of a homology sphere reduces modulo 2 to its Rohlin invariant, compare with Section 9.1 .

10.1. $\mathbb{Z}[\mathbb{Z}]$-homology 4-tori. By $\mathbb{Z}[\mathbb{Z}]$-homology 4-torus we mean a closed oriented smooth spin 4 -manifold $X$ such that $H_{*}(X ; \mathbb{Z})=H_{*}\left(T^{4} ; \mathbb{Z}\right)$ and $H_{*}\left(\tilde{X}_{a} ; \mathbb{Z}\right)=H_{*}\left(T^{3} ; \mathbb{Z}\right)$, where $\tilde{X}_{a}$ is the infinite cyclic cover of $X$ corresponding to a choice of primitive element $a \in H^{1}(X ; \mathbb{Z})$. The intersection form of $X$ on the second cohomology is always isomorphic to the sum of three copies of the hyperbolic 2-form; however, the cup-product on the first cohomology of $X$ may vary. Let $a_{0}, a_{1}, a_{2}$, and $a_{3}$ be a basis in $H^{1}\left(X ; \mathbb{Z}_{2}\right)$ then $\operatorname{det} X=\left(a_{0} \cup a_{1} \cup a_{2} \cup a_{3}\right)[X](\bmod 2)$ is independent of the choice of $a_{0}, a_{1}, a_{2}$, and $a_{3}$ and is called the determinant of $X$. A $\mathbb{Z}[\mathbb{Z}]-$ homology 4 -torus $X$ is called odd if $\operatorname{det} X=1(\bmod 2)$, and is called even otherwise. 
There are any number of ways to construct $\mathbb{Z}[\mathbb{Z}]$-homology 4 -tori; for instance, any homology 3 -torus times a circle is a $\mathbb{Z}[\mathbb{Z}]$-homology 4 -torus. In turn, an ample source of homology 3 -tori is surgery on any 3 -component link with zero framing matrix as in Figure 4 . The $\mathbb{Z}[\mathbb{Z}]$-homology 4-torus $S^{1} \times\left(3 \#\left(S^{1} \times S^{2}\right)\right)$ is even while the torus $T^{4}=S^{1} \times T^{3}$ is odd.

0

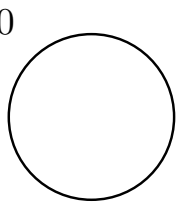

$$
3 \#\left(S^{1} \times S^{2}\right)
$$

0

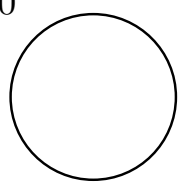

$T^{3}$

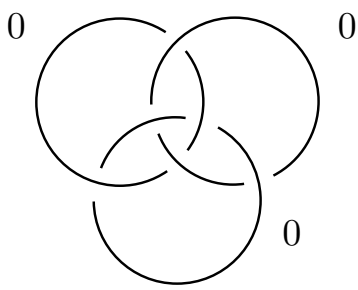

FiguRE 4.

Another interesting family of examples of $\mathbb{Z}[\mathbb{Z}]$-homology 4 -tori is obtained by the following construction. View $T^{4}$ as a trivial $T^{2}$-bundle over $T^{2}$ and consider two embedded disks $D_{a}$ and $D_{b}$ in the base $T^{2}$, see Figure 5 .
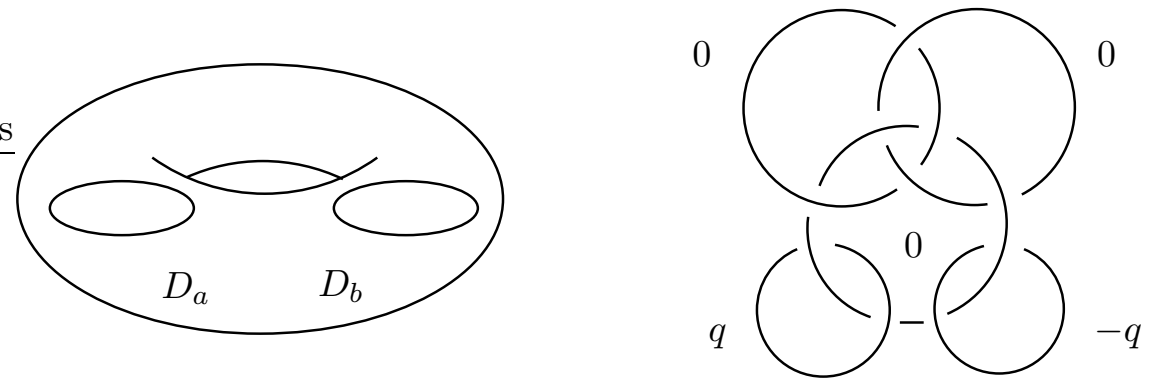

FIGURE 5.

Cut out $D_{a} \times T^{2}$ and $D_{b} \times T^{2}$ and glue them back using automorphisms

$$
\begin{array}{ll}
(a, x, y) \rightarrow\left(a^{q} x^{q-1}, a x, y\right) & \text { on } \partial D_{a} \times T^{2}, \\
(b, x, y) \rightarrow\left(b^{-q} x^{-q-1}, b x, y\right) & \text { on } \partial D_{b} \times T^{2} .
\end{array}
$$

If $q$ is odd, the resulting manifold is an odd $\mathbb{Z}[\mathbb{Z}]$-homology 4 -torus which we denote by $T^{4}(q,-q)$. It is a double branched cover of a log-transform of the Kummer surface with exceptional curves blown down. In fact, $T^{4}(q,-q)=$ $S^{1} \times T^{3}(q,-q)$, where $T^{3}(q,-q)$ is the surgery on the link shown in Figure [5]

It is not necessarily the case that a $\mathbb{Z}[\mathbb{Z}]$-homology 4 -torus is a product with $S^{1}$. Examples of this can arise from the round surgery construction discussed in Section 9.2 Gluing round 2-handles along a collection of embedded disjoint 2 -tori, if done right, will result in a $\mathbb{Z}[\mathbb{Z}]$-homology 4 -torus. 
10.2. The Rohlin invariant. Let $X$ be a $\mathbb{Z}[\mathbb{Z}]$-homology 4 -torus with spin structure $\sigma$, and let $a \in H^{1}(X ; \mathbb{Z})$ be a primitive element such that the cyclic cover $\tilde{X}_{a} \rightarrow X$ corresponding to $a: \pi_{1} X \rightarrow \mathbb{Z}$ has integral homology of $T^{3}$. Let $M \subset X$ be Poincaré dual to $a$ and define the Rohlin invariant

$$
\rho(X, a, \sigma)=\rho(M, \sigma) \quad(\bmod 2)
$$

using the induced spin structure $\sigma$ on $M$. Complete $a$ to a basis $a, x_{1}, x_{2}, x_{3} \in$ $H^{1}\left(X ; \mathbb{Z}_{2}\right)$ and define

$$
\bar{\rho}(X, a)=\sum_{x \in \operatorname{Span}\left\{x_{1}, x_{2}, x_{3}\right\}} \rho(X, a, \sigma+x) .
$$

Theorem 10.1. The invariant $\bar{\rho}(X, a)$ is well defined and $\bar{\rho}(X, a)=\operatorname{det} X$ $(\bmod 2)$.

We refer the reader to our paper [38] for a complete proof of this theorem.

10.3. The Donaldson invariant. Let $X$ be a $\mathbb{Z}[\mathbb{Z}]$-homology 4 -torus, $P \rightarrow X$ an $S O(3)$-bundle with $p_{1}(P)=0$ and $w_{2}(P) \neq 0$, and $\mathcal{G}_{S U(2)}$ the group of automorphisms of $P$ that lift to $S U(2)$. The space $\mathcal{M}(P)$ of ASD connections on $P$ modulo $\mathcal{G}_{S U(2)}$ has formal dimension $-2 p_{1}(P)[X]-$ $3\left(1-b_{1}+b_{+}^{2}\right)(X)=0$. The condition $w_{2}(P) \neq 0$ implies that all connections in $\mathcal{M}(P)$ are irreducible, and it follows from the Chern-Weil theory that all of them are actually flat.

After perturbing the ASD equation $F_{A}^{+}=0$ using a generic admissible perturbation $\sigma$ as described in Section 4.5 we obtain a perturbed moduli space $\mathcal{M}_{\sigma}(P)$ which is a compact oriented manifold of dimension zero, compare with Donaldson 12. The degree zero Donaldson polynomial is defined as

$$
\mathcal{D}_{0}(X, P)=\# \mathcal{M}_{\sigma}(P)
$$

An alternative approach to the definition of $\mathcal{D}_{0}(X, P)$, which we employ in 38, is via $U(2)$ bundles and projectively ASD connections with fixed central part.

In order to state our main result about $\mathcal{D}_{0}(X, P)$, we need to impose two extra hypotheses. One is a mild technical restriction on the bundle $P$ : we assume that there exists $\xi \in H^{1}\left(X ; \mathbb{Z}_{2}\right)$ such that $w_{2}(P) \cup \xi \neq 0$ (note that this condition is automatically satisfied for odd $\mathbb{Z}[\mathbb{Z}]$-homology 4 -tori). The other hypothesis is needed because, at present, we have some unresolved issues in completing the proof of the existence of equivariant perturbations, see Section [10.7. We assume that

$\left(^{*}\right)$ there is an equivariant generic admissible perturbation that makes the ASD moduli space into a smooth 0-manifold.

We are quite confident that the ideas briefly sketched in Section 10.7 will show that hypothesis $(*)$ is always satisfied. 
Theorem 10.2. Under the above restriction on the bundle $P$ (and, for the moment, hypothesis $\left(^{*}\right)$ ), the invariant $\mathcal{D}_{0}(X, P)$ is divisible by four and

$$
\frac{1}{4} \mathcal{D}_{0}(X, P)=\operatorname{det} X \quad(\bmod 2) \text {. }
$$

Note that the formula of Theorem 10.2 need not hold over the integers. For instance, $\mathcal{D}_{0}\left(T^{4}(q,-q), P\right)$ is equal to $\pm 4 q^{2}$ if $P$ is a pull back from $T^{3}(q,-q)$, and to \pm 4 otherwise, see [38. It is also worth mentioning that, in the spirit of Witten's conjecture relating Donaldson and Seiberg-Witten invariants, Theorem 10.2 is consistent with the result of the first author and S. Strle [39] on the $(\bmod 2)$ evaluation of the Seiberg-Witten invariant for homology 4-tori. The main result of this paper is that the Seiberg-Witten invariant of the $\operatorname{Spin}^{c}$ structure associated to a spin structure on a homology 4 -torus $X$ is congruent to $\operatorname{det} X$ modulo 2 .

10.4. A sketch of the proof of Theorem 10.2, Let us assume for the moment that the moduli space $\mathcal{M}(P)$ is non-degenerate. Let $\mathcal{G}_{S O(3)}$ be the full gauge group of automorphisms of $P$, then we have the exact sequence

$$
1 \longrightarrow \mathcal{G}_{S U(2)} \longrightarrow \mathcal{G}_{S O(3)} \longrightarrow H^{1}\left(X ; \mathbb{Z}_{2}\right) \longrightarrow 1
$$

so that the moduli space $\mathcal{M}(P)$ is acted upon by $H^{1}\left(X ; \mathbb{Z}_{2}\right)$ and its quotient is the moduli space $\overline{\mathcal{M}}(P)$ of $\mathcal{G}_{S O(3)}$ equivalence classes of ASD connections on $P$. This action is a complete analogue of the action described in Section 4.6.

The action of $H^{1}\left(X ; \mathbb{Z}_{2}\right)=\left(\mathbb{Z}_{2}\right)^{4}$ is orientation preserving, see Donaldson [12, therefore, in order to compute $\mathcal{D}_{0}(X, P)$, one can count not individual points in $\mathcal{M}(P)$ but rather their orbits. From this point on, we will proceed by showing that there are no orbits of orders one or two, so that $(1 / 4)$. $\mathcal{D}_{0}(X, P)(\bmod 2)$ equals the number of the 4 -orbits (because the eightand the sixteen-orbits do not contribute to the above count).

If the moduli space $\mathcal{M}(P)$ fails to be non-degenerate, it is perturbed using an admissible perturbation $\sigma$ which is equivariant with respect to the action of $H^{1}\left(X ; \mathbb{Z}_{2}\right)$. Achieving non-degeneracy of $\mathcal{M}_{\sigma}(P)$ using equivariant perturbations is not an easy task, see Section 10.7. but once it is done, the above counting argument can be applied to $\mathcal{M}_{\sigma}(P)$ to complete the proof.

10.5. The holonomy correspondence. To do an actual count of the fourorbits, we take advantage of the fact that the connections in $\mathcal{M}(P)$ are flat and hence can be interpreted algebraically using the holonomy map. More precisely, let $w_{2}(P)=w \in H^{2}\left(X ; \mathbb{Z}_{2}\right)$ then the usual holonomy correspondence identifies the moduli space $\overline{\mathcal{M}}(P)$ with a compact subset of the character variety $\mathcal{R}(X, S O(3))$, which we call $\mathcal{R}_{w}(X, S O(3))$. The proper algebraic tool for lifting this correspondence to the covering $\mathcal{M}(P) \rightarrow \overline{\mathcal{M}}(P)$ is projective representations.

A map $\rho: \pi_{1} X \rightarrow S U(2)$ is called a projective representation if $\rho(g h)=$ $c(g, h) \rho(g) \rho(h)$ where $c(g, h)$ belongs to $\mathbb{Z}_{2}=\{ \pm 1\}$ viewed as the center of 
$S U(2)$. The 2 -cocycle $c$ defines a cohomology class $[c] \in H^{2}\left(\pi_{1} X ; \mathbb{Z}_{2}\right)$ such that $[c]=w_{2}(\operatorname{ad} \rho)$. Here, $w_{2}(\operatorname{ad} \rho)$ stands for the second Stiefel-Whitney class of the flat $S O(3)$ bundle with holonomy ad $\rho$, and the above equality makes sense after we identify $H^{2}\left(\pi_{1} X ; \mathbb{Z}_{2}\right)$ as a natural subset of $H^{2}\left(X ; \mathbb{Z}_{2}\right)$. In general, $H^{2}\left(\pi_{1} X ; \mathbb{Z}_{2}\right)$ is not equal to $H^{2}\left(X ; \mathbb{Z}_{2}\right)$; this minor point needs a separate treatment, see 38 .

Define $\mathcal{P} \mathcal{R}_{c}(X ; S U(2))$ to be the set of conjugacy classes of projective representations $\rho$ with fixed $c$. It is acted upon by $H^{1}\left(X ; \mathbb{Z}_{2}\right)$ by the rule $(a, \rho) \rightarrow a \cdot \rho$. According to 36, the holonomy correspondence $\overline{\mathcal{M}}(P) \rightarrow$ $\mathcal{R}_{w}(X, S O(3))$ lifts to a bijective correspondence $\mathcal{M}(P) \rightarrow \mathcal{P} \mathcal{R}_{c}(X, S U(2))$ so that we have the following commutative diagram

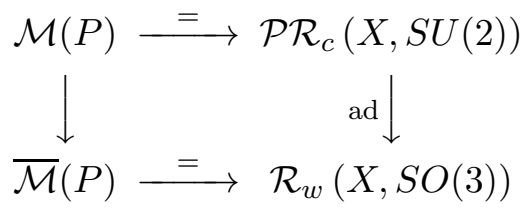

where both vertical arrows are the quotient maps by the action of $H^{1}\left(X ; \mathbb{Z}_{2}\right)$. This interpretation of $\mathcal{M}(P)$ in terms of projective representations allows for a precise description of the four-orbits.

10.6. The four-orbits. Let $a, b \in H^{1}\left(X ; \mathbb{Z}_{2}\right)$ be two different non-trivial elements stabilizing $\rho \in \mathcal{P} \mathcal{R}_{c}(X, S U(2))$. The argument from the proof of Proposition 4.1 when applied simultaneously to $a$ and $b$ shows that, possibly after conjugation, $\operatorname{im} \rho \subset\left(S_{i} \cup j \cdot S_{i}\right) \cap\left(S_{j} \cup k \cdot S_{j}\right)=\{ \pm 1, \pm i, \pm j, \pm k\}$. Equivalently, im ad $\rho \subset \mathbb{Z}_{2} \oplus \mathbb{Z}_{2} \subset S O(3)$ where the latter inclusion is given by $(A, B) \rightarrow A \oplus B \oplus A \cdot B$. The same argument can further be used to show that the action of $H^{1}\left(X ; \mathbb{Z}_{2}\right)$ on $\mathcal{P} \mathcal{R}_{c}(X, S U(2))$ does not have orbits of orders one or two.

Thus the set of the four-orbits is in a bijective correspondence with the set of representations ad $\rho: \pi_{1} X \rightarrow S O(3)$ which factor through a subgroup $\mathbb{Z}_{2} \oplus \mathbb{Z}_{2}$ of $S O(3)$, modulo $S O(3)$ conjugation. Every such a representation induces representations $\alpha, \beta: \pi_{1} X \rightarrow \mathbb{Z}_{2}$, which can be viewed as cohomology classes $\alpha, \beta \in H^{1}\left(X ; \mathbb{Z}_{2}\right)$. This identifies the set of the 4 -orbits with $\Lambda_{0}^{2} H^{1}\left(X ; \mathbb{Z}_{2}\right)$, the set of decomposable elements in the second exterior power of $H^{1}\left(X ; \mathbb{Z}_{2}\right)$. A straightforward calculation gives $w_{2}(\operatorname{ad} \rho)=\alpha \cup \beta \in H^{2}\left(X ; \mathbb{Z}_{2}\right)$, hence the set of the four-orbits with fixed $w$ can be identified with the preimage of $w$ under the map

$$
\cup: \Lambda_{0}^{2} H^{1}\left(X ; \mathbb{Z}_{2}\right) \longrightarrow H^{2}\left(X ; \mathbb{Z}_{2}\right) \text {. }
$$

It is an exercise in algebraic topology to show that, under the hypotheses of Theorem 10.2 the above map is a bijection if $\operatorname{det} X$ is odd and is zero if $\operatorname{det} X$ is even.

10.7. Perturbations. The above argument only applies as stated if the moduli space $\mathcal{M}(P)$ is non-degenerate, otherwise, $\mathcal{M}(P)$ needs to be perturbed first. It turns out that the four-orbits are always non-degenerate and 
remain such after a small enough perturbation, so the argument of Section 10.6 counting the four-orbits goes unchanged. Thus the eight- and sixteenorbits are the only ones that are perturbed. As of this writing, some details of what is briefly described below remain to be completely verified.

We define our perturbations $\sigma$ using holonomy around loops $\psi_{k}$ as in Section 4.5. In the equivariant setting, we have to address two new issues. First, we need to make $\sigma$ equivariant with respect to the $H^{1}\left(X ; \mathbb{Z}_{2}\right)$ action; this is done by requiring that $0=\left[\psi_{k}\right] \in H_{1}\left(X ; \mathbb{Z}_{2}\right)$. Next, we need to show that these equivariant perturbations are generic, that is, there are enough

of them to make $\mathcal{M}_{\sigma}(P)$ non-degenerate. Perturbations along disjoint loops as in Section 4.5] are sufficient if the action of $H^{1}\left(X ; \mathbb{Z}_{2}\right)$ is free; otherwise, one may need more general perturbations. We refer the reader to the forthcoming revision of [38] for all the details.

It is worth mentioning that, since the quotient space of $\mathcal{M}(P)$ by the $H^{1}\left(X ; \mathbb{Z}_{2}\right)$ action is the $S O(3)$ moduli space $\overline{\mathcal{M}}(P)$, the equivariant perturbation theory described above is essentially the $S O(3)$ perturbation theory.

\section{REFERENCES}

[1] Akbulut, S. A fake 4-manifold. Four-manifold theory, 75-141, Contemp. Math. 35, Amer. Math. Soc., Providence, RI, 1984.

[2] Akbulut, S. A fake compact contractible 4-manifold. J. Diff. Geom. 33 (1991), 335356.

[3] Akbulut, S. and McCarthy, J. Casson's invariant for oriented homology 3-spheres. An exposition. Mathematical Notes, 36. Princeton University Press, Princeton, NJ, 1990.

[4] Asimov, D. Round handles and non-singular Morse-Smale flows. Ann. of Math. (2) 102 (1975), 41-54.

[5] Atiyah, M., Patodi, V. and Singer, I. Spectral asymmetry and Riemannian geometry.I. Math. Proc. Cambridge. Philos. Soc. 77 (1975), 43-69.

[6] Boyer, S. and Lines, D. Surgery formulae for Casson's invariant and extensions to homology lens spaces. J. Reine Angew. Math. 405 (1990), 181-220.

[7] Boyer, S. and Nicas, A. Varieties of group representations and Casson's invariant for rational homology 3-spheres. Trans. Amer. Math. Soc. 322 (1990), 507-522.

[8] Braam, P. and Donaldson, S. Floer's work on instanton homology, knots and surgery. The Floer memorial volume, 195-256, Progr. Math. 133, Birkhäuser, Basel, 1995.

[9] Cappell, S., Lee, R. and Miller, E. Self-adjoint elliptic operators and manifold decompositions. III. Determinant line bundles and Lagrangian intersection. Comm. Pure Appl. Math. 52 (1999), 543-611.

[10] Cappell, S. and Shaneson, J. Some new four-manifolds. Ann. of Math. 104 (1976), $61-72$.

[11] Collin, O. and Saveliev, N. Equivariant Casson invariants via gauge theory. J. Reine Angew. Math. 541 (2001), 143-169.

[12] Donaldson, S. The orientation of Yang-Mills moduli spaces and 4-manifold topology. J. Diff. Geom. 26 (1987), 397-428.

[13] Donaldson, S. and Kronheimer, P. The Geometry of Four-Manifolds. Oxford University Press, 1990.

[14] Fintushel, R. and Stern, R. An exotic free involution on $S^{4}$. Ann. of Math. (2) 113 (1981), 357-365. 
[15] Fintushel, R. and Stern, R. Instanton homology of Seifert fibred homology three spheres. Proc. London Math. Soc. (3) 61 (1990), 109-137.

[16] Floer, A. An instanton-invariant of 3-manifolds. Comm. Math. Phys. 118 (1988), 215-240.

[17] Floer, A. Instanton homology and Dehn surgery. The Floer memorial volume, 77-97, Progr. Math. 133, Birkhäuser, Basel, 1995.

[18] Frøyshov, K. Equivariant aspects of Yang-Mills Floer theory. Topology 41 (2002), 525-552.

[19] Fukumoto, Y. and Furuta, M. Homology 3-spheres bounding acyclic 4-manifolds. Math. Res. Lett. 7 (2000), 757-766.

[20] Fukumoto, Y., Furuta, M. and Ue, M. W-invariants and Neumann-Siebenmann invariants for Seifert homology 3-spheres. Topology Appl. 116 (2001), 333-369.

[21] Furuta, M. and Ohta, H. Differentiable structures on punctured 4-manifolds. Topology Appl. 51 (1993), 291-301.

[22] Galewski, D. and Stern, R. Classification of simplicial triangulations of topological manifolds. Ann. of Math. (2), 111 (1980), 1-34.

[23] Gompf, R. and Stipsicz, A. 4-manifolds and Kirby calculus. Graduate Studies in Mathematics, 20. Amer. Math. Soc., Providence, RI, 1999.

[24] Herald, C. Flat connections, the Alexander invariant, and Casson's invariant. Comm. Anal. Geom. 5 (1997), 93-120.

[25] Herald, C. Legendrian cobordism and Chern-Simons theory on 3-manifolds with boundary. Comm. Anal. Geom. 2 (1994), 337-413.

[26] C. Herald, Transversality for equivariant gradient systems and gauge theory on 3manifolds Adv. Math. (to appear)

[27] Kaplan, S. Constructing framed 4-manifolds with given almost framed boundaries. Trans. Amer. Math. Soc. 254 (1979), 237-263.

[28] Kirby, R. and Taylor, L. A survey of 4-manifolds through the eyes of surgery. Surveys on surgery theory, Vol. 2, 387-421. Ann. of Math. Stud. 149, Princeton University Press, Princeton, NJ, 2001. URL: http://arxiv.org/math.GT/9803101

[29] Levine, J. Polynomial invariants of knots of codimension two. Ann. of Math. (2) 84 (1966), 537-554.

[30] Matumoto, T. Triangulation of manifolds. Algebraic and geometric topology, Part 2, 3-6, Proc. Sympos. Pure Math., XXXII, Amer. Math. Soc., Providence, RI, 1978.

[31] Morgan, J., Mrowka, T. and Ruberman, D. The $L^{2}$-moduli space and a vanishing theorem for Donaldson polynomial invariants. International Press, Cambridge, MA, 1994.

[32] Neumann, W. An invariant of plumbed homology spheres. Topology Symposium, Siegen 1979, 125-144, Lect. Notes in Math., 788, Springer, Berlin, 1980.

[33] Nicolaescu, L. Notes on Seiberg-Witten theory. Graduate Studies in Mathematics, 28. Amer. Mah. Soc., Providence, RI, 2000.

[34] Nicolaescu, L. http://www.nd.edu/ lnicolae/OT.pdf

[35] Ruberman, D. Doubly slice knots and the Casson-Gordon invariants. Trans. Amer. Math. Soc. 279 (1983), 569-588.

[36] Ruberman, D. and Saveliev, N. Rohlin's invariant and gauge theory I. Homology 3-tori. Comment. Math. Helv. 79 (2004), 618-646. URL: http://arxiv.org/math.GT/0302131

[37] Ruberman, D. and Saveliev, N. Rohlin's invariant and gauge theory II. Mapping tori. Geom. Topol. 8 (2004), 35-76. URL: http://arXiv.org/math.GT/0306188

[38] Ruberman, D. and Saveliev, N. Rohlin's invariant and gauge theory III. Homology 4-tori (submitted). URL: http://arXiv.org/math.GT/0404162

[39] Ruberman, D. and Strle, S. Mod 2 Seiberg-Witten invariants of homology tori. Math. Res. Lett. 7 (2000), 789-799. 
[40] Saveliev, N. Floer homology of Brieskorn homology spheres. J. Diff. Geom. 53 (1999), $15-87$.

[41] Saveliev, N. Fukumoto-Furuta invariants of plumbed homology 3-spheres. Pacific J. Math. 205 (2002), 465-490.

[42] Saveliev, N. Invariants for homology 3-spheres. Encyclopaedia of Math. Sciences, 140. Springer, Berlin, 2002.

[43] Saveliev, N. Lectures on the topology of 3-manifolds. An introduction to the Casson invariant. Walter de Gruyter \& Co., Berlin, 1999.

[44] Saveliev, N. A note on Akbulut corks. Math. Res. Lett. 10 (2003), 777-785.

[45] Saveliev, N. Representation spaces of Seifert fibered homology spheres. Topology Appl. 126 (2002), 49-61.

[46] Shaneson, J. Wall's surgery obstruction groups for $G \times \mathbb{Z}$. Ann. of Math. (2) 90 (1969), 296-334.

[47] Siebenmann, L. On vanishing of the Rohlin invariant and nonfinitely amphicheiral homology 3-spheres. Topology Symposium, Siegen 1979, 172-222, Lect. Notes in Math. 788, Springer, Berlin, 1980.

[48] Taubes, C. Casson's invariant and gauge theory. J. Diff. Geom. 31 (1990), 547-599.

[49] Taubes, C. Gauge theory on asymptotically periodic 4-manifolds. J. Diff. Geom. 25 (1987), 363-430.

[50] Viro, O. Branched coverings of manifolds with boundary and link invariants. Math. USSR Izvestia 7 (1973), 1239-1256.

[51] Walker, K. An extension of Casson's invariant. Ann. of Math. Studies, 126. Princeton University Press, Princeton, NJ, 1992.

[52] Wall, C. T. C. Surgery on Compact Manifolds. Academic Press, London-New York, 1970.

Department of Mathematics, MS 050, Brandeis University, Waltham, MA 02454

E-mail address: ruberman@brandeis.edu

Department of Mathematics, University of Miami, Box 249085, Coral Gables, FL 33124

E-mail address: saveliev@math.miami.edu 